\title{
Gas Phase Silver Thermochemistry from First
}

\section{Principles.}

Irina Minenkova, ${ }^{\ddagger}$, Valery V. Sliznev, ${ }^{\neq}$Luigi Cavallo $^{\dagger}$ and Yury Minenkov ${ }^{\ddagger} * *$

‡ Moscow Institute of Physics and Technology, Institutskiy Pereulok 9, Dolgoprudny, Moscow

Region 141700, Russian Federation

$\S$ Frumkin Institute of Physical Chemistry and Electrochemistry, Russian Academy of Sciences, Leninskii pr. 31, Moscow 119071, Russian Federation

\# Ivanovo State University of Chemistry and Technology, Research Institute for Thermodynamics and Kinetics of Chemical Processes, 153460 Ivanovo, Russian Federation

† KAUST Catalysis Center (KCC), King Abdullah University of Science and Technology, Thuwal-23955-6900, Saudi Arabia

ABSTRACT. Domain based local pair natural orbital coupled cluster approach with single, double, and perturbative triple excitations, DLPNO-CCSD(T), has been applied within a framework of a reduced version of the reaction-based Feller-Peterson-Dixon (FPD) scheme to predict gas phase heats of formation and absolute entropies of silver inorganic and organometallic compounds. First, we evaluated all existing experimental data currently limited by thermodynamic functions of 10 silver substances ( $\mathrm{AgH}, \mathrm{AgF}, \mathrm{AgBr}, \mathrm{AgI}, \mathrm{Ag}_{2}, \mathrm{Ag}_{2} \mathrm{~S}, \mathrm{Ag}_{2} \mathrm{Se}, \mathrm{Ag}_{2} \mathrm{Te}, \mathrm{AgCN}, \mathrm{AgPO}_{2}$ ). The mean 
average deviation between computed and experimental heats of formation was found to be 1.9 $\mathrm{kcal} / \mathrm{mol}$. Notably, all predicted heats of formations turned out to be within the error bounds of their experimental counterparts. Second, we predicted heats of formation and entropies for additional 90 silver species with no experimental data available, substantially enriching silver thermochemistry. Combination of gas phase heats of formation $\Delta \mathrm{H}_{\mathrm{f}}$ and entropies $\mathrm{S}^{\circ}$ of $\mathrm{AgNO}_{2}$, $\mathrm{AgSCN}, \mathrm{Ag}_{2} \mathrm{SO}_{4}$, and $\mathrm{Ag}_{2} \mathrm{SeO}_{4}$ obtained in this work, with respective solid-state information, resulted in accurate sublimation thermochemistry of these compounds. Complementation of predicted $\Delta \mathrm{H}_{\mathrm{f}}$ with heats of formation of some neutrals and positive ions produced 33 silver bond strengths of high reliability. Obtained thermochemical data are promising for developing the concepts of silver chemistry. In addition, derived heats of formation and bond dissociation enthalpies, due to their high diversity, are to be relevant for testing and training of computational chemistry methods.

KEYWORDS: Transition Metals, Silver, Heats of Formation, Thermochemistry, DLPNO$\operatorname{CCSD}(\mathrm{T})$, Silver Database

\section{Introduction}

Inorganic silver salts feature a number of interesting properties, forming a basis for numerous industrial applications. ${ }^{1,2}$ Due to their light sensitivity, silver halides have been utilized in photography ${ }^{3-5}$ since the beginning of $19^{\text {th }}$ century. As silver cation possesses antimicrobial activity, many silver salts are used as antiseptics ( $\mathrm{AgCl}, \mathrm{AgI})$ and water purification agents (AgF). In chemical industry and fine organic synthesis, many silver compounds are exploited as Lewis acids, oxidizers $\left(\mathrm{AgNO}_{2}\right)$, fluorinating agents $(\mathrm{AgF})$ and catalysts $\left(\mathrm{AgBr}, \mathrm{Ag}_{2} \mathrm{~S}, \mathrm{AgNO}_{2}\right.$, etc.). ${ }^{6-18}$ 
A few other applications include cloud seeding agents (AgI), silver plating agents $\left(\mathrm{Ag}_{2} \mathrm{SO}_{4}\right.$, $\mathrm{AgCN})$ explosives (AgCNO), semiconductors $\left(\mathrm{Ag}_{2} \mathrm{Se}, \mathrm{AgBr}, \mathrm{Ag}_{2} \mathrm{Te}\right)$ and electronics-related materials. ${ }^{19-21}$ To explain physical and chemical properties of silver species, an information about their gas phase accurate thermodynamic functions is essential. Perhaps surprisingly, but a careful analysis of (online) databases as such as the NIST-JANAF tables, ${ }^{22-24}$ the Glushko/Gurvich compilations, ${ }^{25-28}$ the handbooks of Krasnov $^{29}$ and the third millennium thermodynamic database $(\mathrm{TMTD})^{30}$ has revealed only a few silver species for which gas phase thermodynamic functions are available. In particular, formation enthalpies $\left(\Delta \mathrm{H}_{\mathrm{f}}\right)$ and molar entropies $\left(\mathrm{S}^{\circ}\right)$ were derived from experimental measurements only for $\mathrm{AgH}, \mathrm{AgF}, \mathrm{AgCl}, \mathrm{AgBr}, \mathrm{AgI}, \mathrm{Ag}_{2}, \mathrm{Ag}_{2} \mathrm{~S}, \mathrm{Ag}_{2} \mathrm{Se}, \mathrm{Ag}_{2} \mathrm{Te}$, and $\mathrm{AgPO}_{2}$.

With this work, we aim to fill the gap and derive accurate thermodynamic functions for a number of silver species using computational chemistry as a tool. Due to a systematic increase in computer power and advances in method development, contemporary computational chemistry approximations are becoming a valuable source of accurate thermodynamic data, ${ }^{31-42}$ quite often outperforming experimental measurements, especially bearing in mind high economic costs associated with the latter. The latest revisions of composite schemes, such as the Weizman-n theories ${ }^{43,44}$ the Gaussian- $4,{ }^{45}$ correlation consistent composite approach $(\mathrm{ccCA})^{46}$ and the FellerPeterson-Dixon (FPD) protocol, ${ }^{32,36,47,48}$ are all capable of predicting gas phase formation enthalpies of organic and inorganic species with the accuracy of $1-2 \mathrm{kcal} / \mathrm{mol}$, if multireference effects are insignificant. However, the coupled cluster method with single, double, and perturbative triple excitations, $\operatorname{CCSD}(\mathrm{T}),{ }^{49}$ which is the electronic structure method at the root of composite schemes, has poor $\mathrm{N}^{7}$ scalability, where $\mathrm{N}$ is the system size, and converges slowly with respect to the basis set size. This makes accurate calculations feasible only for molecules of a 
dozen heavy atoms, and only for the groups having access to large computational facilities. An advent of local $\operatorname{CCSD}(\mathrm{T})$ methods, ${ }^{50-55}$ in particular, DLPNO-CCSD(T), ${ }^{54-56}$ accelerated by resolution of identity approximation and efficient algorithms for two electron transformations ${ }^{50,57-}$ ${ }^{59}$ resulted in much faster calculations increasing their role in chemical thermodynamics. A few studies performed on organic ${ }^{60-62}$ and inorganic species ${ }^{63,64}$ indicated that resulting DLPNO$\operatorname{CCSD}(\mathrm{T})$ heats of formations are practically identical to their canonical $\operatorname{CCSD}(\mathrm{T})$ counterparts.

For these reasons, we utilized a reduced version of reaction-based FPD protocol, successfully used by us earlier to predict transition metal heats of formation poorly defined in NIST-JANAF tables, ${ }^{63}$ to predict heats of formation and entropies for a number of silver species, composing a new silver database. The reduced FPD protocol includes correlation of sub-valence electrons, complete basis set (CBS) extrapolated aug-cc-pwCVTZ/aug-cc-pwCVQZ DLPNO$\operatorname{CCSD}(\mathrm{T})$ energies and density functional theory (DFT) $)^{65,66}$ geometries and harmonic frequencies. Apart from direct application in industrial thermodynamic modeling and thermal engineering, this kind of integrated experimental-theoretical databases are highly relevant for development and testing of semiempirical neglect diatomic differential overlap (NDDO) ${ }^{67,68}$ and DFT (e.g. as B3LYP ${ }^{69-71}$ ) methods as we tried to achieve the highest diversity on our dataset, in particular by the inclusion of unstable compounds. Finally, thermodynamic functions of individual gas phase compounds, as well as chemical reaction, heats/Gibbs free energy changes derived from them are the source of reliable reference data to benchmark the computational chemistry methods..$^{60,63,64,72-}$ 78

\section{Computational Details}

\subsection{Calculation of Reference Values}




\subsubsection{Geometry Optimization and Calculation of Harmonic Frequencies}

All geometry optimizations have been performed with the hybrid GGA PBE0 $0^{79-81}$ functional as implemented in the Gaussian $09^{82}$ suite of programs. The PBE0 functional was chosen due to its good performance in reproducing of molecular structures of transition metal complexes. $^{83-86}$ The default values for the self-consistent-field and geometry optimization convergence criteria were adopted. The numerical integration of the exchange-correlation (XC) terms was performed using the tighter-than-default "ultrafine" option (pruned, 99 radial shells and 590 angular points per shell) to eliminate the potential numerical noise in the energy derivatives. Geometries were characterized as true energy minima by the eigenvalues of the analytically calculated Hessian matrix. Translational, rotational, and vibrational partition functions for thermal corrections to give total enthalpies and entropies were computed within the ideal-gas, rigid-rotor, and harmonic oscillator approximations following standard procedures. ${ }^{87,88}$ The temperature was set to $298.15 \mathrm{~K}$ in all cases. All harmonic frequencies were scaled prior calculating of the harmonic oscillator partition functions. The scaling factor of 0.9720 was employed as recommended in ref. ${ }^{89}$ for calculation of enthalpic correction at PBE0/6-311+G(2df,p) approximation. This scaling factor is reasonably close to 0.9834 recommended by Martin and co-workers for calculation ZPE and PBE0/aug-cc-pVT $(+\mathrm{d}) \mathrm{Z}$ method. ${ }^{90}$ An effect of scaling the frequencies on resulting reaction enthalpies and entropies turned out to be insignificant, vide infra.

All electron triple- $\zeta$ correlation-consistent basis sets augmented with diffuse functions (aug-cc-pVTZ) $)^{91,92}$ have been applied to describe the electronic shells of hydrogen, carbon, nitrogen, oxygen, fluorine, silicon, phosphorus, sulfur and chlorine atoms. Outer electronic shells of germanium, arsenic, tin, antimony, selenium, bromine, tellurium, silver and iodine atoms have been described with aug-cc-pVTZ-PP of Peterson and co-workers. ${ }^{93-96}$ Fully relativistic effective 
core potentials (ECP) have been utilized to describe the 10 core electrons of Ge and As, ${ }^{97}$ and Se and $\mathrm{Br},{ }^{94}$ and the 28 core electrons of $\mathrm{Sn}$ and $\mathrm{Sb},{ }^{97} \mathrm{Te},{ }^{94} \mathrm{Ag},{ }^{98}$ and $\mathrm{I} .{ }^{96}$ These basis sets and ECPs were only used to obtain equilibrium molecular geometries and harmonic frequencies. The basis sets/ECPs utilized for SP energies are defined in the next Section.

If several local potential energy surface (PES) minima were possible for some molecules, the most stable structure was identified by geometry optimization of all possible conformers and isomers and comparing their PBE0/aug-cc-pVTZ(-PP) electronic energies. A similar strategy has been employed to check the relative energies of higher spin states. In all cases, the singlet state turned out to be the most stable form.

\subsubsection{Single Point Energy Evaluation}

The DLPNO-CCSD(T) method of Neese and co-workers ${ }^{54-56}$ as implemented in ORCA 4.0 99 suite of programs was applied for single-point energy evaluations. Tighter than the default “TightPNO" DLPNO settings (TCutPairs $=10^{-5}$, TCutPNO $=10^{-7}$, and TCutMKN $=10^{-3}$ ) were used, as recommended, for applications where the most accurate values are targeted. ${ }^{100}$ Apart from hydrogen, for all atoms the sub-valence electrons were correlated as it was shown to be critically important for accurate formation enthalpies of some compounds. ${ }^{48,63,64,72,101-108}$ The following triple and quadruple- $\zeta$ correlation consistent basis sets augmented with diffuse functions were used in the present work. Dunning's aug-cc-pVnZ basis sets were applied to describe the hydrogen atom. ${ }^{91}$ Carbon, nitrogen, oxygen, fluoride, silicon, phosphorus, sulfur and chlorine were described with the correlation consistent weighted core valence aug-cc-pwCVnZ basis sets of Peterson and Dunning. ${ }^{109}$ Germanium, arsenic, selenium, bromine, tin, antimony, tellurium and iodine atoms have been described by the correlation consistent weighted core valence aug-cc-pwCVnZ-PP basis 
sets of Peterson and Yousaf. ${ }^{110}$ Finally, the correlation consistent weighted core valence aug-ccpwCVnZ-PP basis sets of Peterson and Puzzarini were utilized to describe the silver atom. ${ }^{95}$ Fully relativistic ECPs identical to those described in Section 2.1.1 have been utilized to describe the core electrons of Ge, As, $\mathrm{Se}, \mathrm{Br}, \mathrm{Sn}, \mathrm{Sb}, \mathrm{Te}, \mathrm{Ag}$, and I. The correlation fitting basis sets necessary for the resolution of identity approximation as part of the DLPNO scheme, were generated by AutoAux routine developed by Neese and co-workers as a part of the ORCA 4 code. ${ }^{111} \mathrm{~A}$ few diagnostic values have been monitored to judge the applicability of $\operatorname{CCSD}(\mathrm{T})$ approach to study the molecules in this work. First, an Euclidian norm of the vector of $t_{1}$ amplitudes in the coupled cluster singles and doubles wave function normalized by the number of correlated electrons, $\mathrm{T}_{1}$ diagnostics, ${ }^{112}$ was utilized as an indicator of the quality of the reference Hartree-Fock orbitals. Second, the largest absolute double excitation amplitude value, $\mathrm{T}_{2}$, was used as an indicator of potential multireference character of the systems, ${ }^{77,113,114}$ see the SI. For the sake of simplicity, we only report here that in all the cases the $T_{1}$ and $T_{2}$ diagnostics values turned out to be reasonably low, indicating good quality of the reference HF wave function $\left(T_{1}\right)$ and no signs of substantial multiconfigurational character of the systems $\left(\mathrm{T}_{2}\right)$.

To account for the basis set incompleteness effects, we applied the extrapolation schemes for HF and DLPNO-CCSD(T) correlation energies of each molecule suggested by Helgaker and coworkers, ${ }^{115-117}$ see Eqs 1. and 2. For two adjacent triple and quadruple- $\zeta$ basis sets:

$$
\begin{aligned}
& E_{H F}^{n}=E_{H F}^{\infty}+\alpha e^{-1.63 n} \\
& E_{\text {corl }}^{n}=E_{\text {corl }}^{\infty}+\beta n^{-3}
\end{aligned}
$$

Where $E_{H F}^{n}$ and $E_{\text {corl }}^{n}$ are the HF and correlation energies calculated with the triple- $(\mathrm{n}=3)$ and quadruple- $\zeta(\mathrm{n}=4)$ basis sets; $E_{H F}^{\infty} / E_{c o r l}^{\infty}$ are the HF and correlation energies at the complete basis set (CBS) limit; $\alpha / \beta$ are parameters to be obtained from a system of the two equations. The total 
absolute enthalpy at CBS limit for the molecule A necessary to evaluate the reaction enthalpies was computed via Eq. 3:

$$
H_{C C S D(T)}^{0}=E_{H F}^{\infty}(A)+E_{\text {corl }}^{\infty}(A)+H_{\text {corr }}^{P B E 0}(A)
$$

where $H_{c o r r}^{P B E 0}$ is the correction to the electronic energy (including both zero-point energy (ZPE) and a thermal correction) to arrive to the enthalpy obtained with standard harmonic oscillator/rigid rotor/ideal gas approximation, see Section 2.1.1.

\subsubsection{Reference Heats of Formation}

Accurate reference formation enthalpies are critical for the reaction-based approach ${ }^{118}$ applied in this work to derive the heats of formation and silver bond strengths, see Sections 3.1 for details. Only the most reliable experimental values have been carefully selected and collected in Table S1. First, $\Delta \mathrm{H}_{\mathrm{f}}(298)$ of $\mathrm{H}^{-}, \mathrm{H}^{+}, \mathrm{OH}^{-}, \mathrm{CN}^{-}, \mathrm{CNO}^{-}, \mathrm{NCO}^{-}, \mathrm{H}_{2} \mathrm{O}, \mathrm{NH}_{2}^{-}, \mathrm{CH}_{4}, \mathrm{CH}_{3}{ }^{-}, \mathrm{CH}_{2} \mathrm{ClBr}, \mathrm{CH}_{2} \mathrm{BrF}, \mathrm{CO}$, $\mathrm{CO}_{2}, \mathrm{NH}_{3}, \mathrm{I}_{2}, \mathrm{HF}, \mathrm{HCl}, \mathrm{HBr}, \mathrm{HI}, \mathrm{ClF}, \mathrm{BrCl}, \mathrm{ICl}, \mathrm{IBr}, \mathrm{NO}_{2}{ }^{-}, \mathrm{NO}_{3}{ }^{-}, \mathrm{Cl}_{2}, \mathrm{~N}_{2}, \mathrm{C}_{2} \mathrm{H}_{2}, \mathrm{Br}_{2}, \mathrm{H}_{2} \mathrm{O}_{2}, \mathrm{~F}^{-}, \mathrm{Cl}^{-}$ , $\mathrm{Br}^{-}, \mathrm{I}^{-}$have been taken from ATcT compilation of Ruscic and co-workers ${ }^{119}$ as these values are considered to be most accurate, reliable, consistent, and these are generally accepted across the physical chemistry community. Second, heats of formation of $\mathrm{H}_{2}, \mathrm{~F}_{2}, \mathrm{~N}_{2}, \mathrm{Cl}_{2}, \mathrm{He}, \mathrm{Ne}, \mathrm{Ar}, \mathrm{Kr}$, and $\mathrm{Xe}$ are all $0 \mathrm{kcal} / \mathrm{mol}$ by definition. Third, atomic formation enthalpies of $\mathrm{Be}, \mathrm{Ca}, \mathrm{Mg}, \mathrm{Sr}, \mathrm{Ba}, \mathrm{Zn}$, $\mathrm{Hg}$ have all been taken from NIST-JANAF thermochemical tables. ${ }^{22}$ All these values are consistent with the data from a compilation of Gurvich et al. ${ }^{25}$ and have been utilized to derive accurate formation enthalpies via a reduced version of reaction-based Feller-Peterson-Dixon (FPD) protocol. ${ }^{120,121}$ Cadmium formation enthalpy has been adopted from CODATA compilation $^{122}$ and it agrees well with data collected by Gurvich et al. ${ }^{25}$ Then, for all other reference species only those experimental heats of formation have been selected that are confirmed by either 
other experimental measurements or by high-quality $\operatorname{CCSD}(\mathrm{T})$ calculations. $\mathrm{AgCl} \Delta \mathrm{H}_{\mathrm{f}}(298)$ of 22.0 $\pm 0.3 \mathrm{kcal} / \mathrm{mol}$ measured by Hildenbrand et al. ${ }^{123}$ was used as a reference for all silver complexes, see SI for details.

\section{Results and Discussion}

The Results and Discussion section is organized into two main parts. In the first part, Section 3.1, we discuss the calculated gas-phase heats of formation and absolute entropies of 100 silver species. We start (Section 3.1.1) from an analysis of gas-phase heats of formation and entropies of a few selected silver compounds for which the literature values are available. Comments on the accuracy of both our theoretical values and their experimental counterparts are given. Then, we present (Section 3. 1.2) the predicted gas phase enthalpies and entropies of 90 silver complexes for which no literature data are reported up to date. In the second part, Section 3.2, we utilize the formation enthalpies $\Delta \mathrm{H}_{\mathrm{f}}$ and entropies $\mathrm{S}^{\circ}$ obtained in this work to derive useful thermodynamic quantities. In Section 3.2.1, using available reference solid-state formation enthalpies and absolute entropies for a few silver species, we retrieve respective thermodynamic functions of sublimation. Then, in Section 3.2.2 taking advantage of additional experimental heats of formation, we extract 33 silver bond strengths.

\subsection{Predicted Enthalpies of Formation and Entropies}

The heats of formation have been obtained by reaction-based approach, pioneered by Pople and co-workers ${ }^{118}$ for bond separation reactions to converge the results at smaller basis set due to compensation of errors, within a reduced version of Feller-Peterson-Dixon (FPD) formalism. ${ }^{32,36,47,48,124-126}$ Although we did not use bond-separation reactions in this work, we 
expect that cancellation of errors in chemical reactions utilized in this work is substantially larger than in corresponding atomization energies. This approximation can be illustrated with the $\operatorname{Ag}_{2} \mathrm{~S}$ molecule for which no gas phase experimental formation enthalpy is known. To derive its formation enthalpy we compose a chemical reaction in such a way that apart from $\mathrm{Ag}_{2} \mathrm{~S}$, it contains only the substances for which the experimental formation enthalpies are known: $\mathrm{HCl}, \mathrm{AgCl}$, and $\mathrm{H}_{2} \mathrm{~S}$, see Eq. 4.

$\mathrm{Ag}_{2} \mathrm{~S}+2 \mathrm{HCl} \rightarrow 2 \mathrm{AgCl}+\mathrm{H}_{2} \mathrm{~S}, \Delta \mathrm{H}_{\mathrm{rxn}}(298 \mathrm{~K})$

Then, the formation enthalpy of $\mathrm{Ag}_{2} \mathrm{~S}$ was calculated via the experimental formation enthalpies of $\mathrm{AgCl}, \mathrm{HCl}$ and $\mathrm{H}_{2} \mathrm{~S}$ and theoretically calculated enthalpy of equation 4. The absolute gas phase entropies have been calculated within the ideal gas - rigid rotor - harmonic oscillator approximation as outlined in Section 2.1.1.

\subsubsection{Comparing with Existing Gas Phase Data}

As accurate gas phase molecular geometries are available for some silver species studied in this work, we compared predicted silver internuclear distances to their experimental counterparts. The results are summarized in Table 1. In general, good agreement has been observed between calculated and reference experimental internuclear distances corroborating our earlier findings on reasonable performance of PBE0 for predicting molecular geometries. ${ }^{86,127}$ The deviations less than $0.02 \AA$ have been detected for $\mathrm{AgH}, \mathrm{AgF}, \mathrm{AgCl}, \mathrm{AgBr}$. $\mathrm{AgI}, \mathrm{AgCN}, \mathrm{ClAgNH}_{3}, \mathrm{IAgPH}_{3}$, and $\mathrm{IAgNH}_{3}$ species.

The calculated PBE0 bond distance Ag-Ag turned out to be $0.029 \AA$ A longer than corresponding experimental value. In IAgCO molecule, calculated Ag-I distance is only $0.01 \AA$ longer comparing 
to its experimental counterpart. ${ }^{128}$ High-level CCSD(T)-F12 calculations performed by Stephens et al. ${ }^{128}$ resulted in $\mathrm{Ag}-\mathrm{I}$ distance only $0.005 \AA$ shorter comparing to experimental $\mathrm{r}_{\mathrm{s}}$ value. Our calculated Ag $-\mathrm{C}$ distance turned out to be $0.045 \AA$ shorter comparing to experimentally measured ${ }^{128} \mathrm{r}_{\mathrm{s}}$ value of $2.053(2) \AA$. The $\mathrm{CCSD}(\mathrm{T})-\mathrm{F} 12$ calculations in ref. ${ }^{128}$ predict $\mathrm{Ag}-\mathrm{C}$ distance to be only $0.015 \AA$ shorter comparing to $\mathrm{r}_{\mathrm{s}}$. Hence, too long $\mathrm{Ag}-\mathrm{C}$ distance in IAgCO obtained in this work is likely due to using of PBE0 rather than due to different physical meaning between calculated $R_{e}$ and experimentally measured $r_{s}$ distances.

To check whether non-negligible discrepancies between PBE0 and experimental geometries might result in significant inaccuracies in reaction energies, we calculated DLPNO-CCSD(T)/augccpwCVTZ(-PP) energies on experimental geometries as well, see Table S2. Table S2 suggests that on average $0.05 \mathrm{kcal} / \mathrm{mol}$ error appears due to using PBE0 instead of experimental geometries. The largest error of $0.12 \mathrm{kcal} / \mathrm{mol}$ was found for $\mathrm{IAgCO}$ molecule discussed above. Hence, we expect that using the PBE0 method does not introduce any significant error in predicted heats of formation. Apparently, the potential energy surfaces (PESs) of investigated molecules are very flat, and deviations of a few hundredths of an angstrom in internuclear distances correspond to very small energy differences.

Table 1. Comparison of predicted internuclear distances formed by silver to their experimental counterparts in selected species studied in this work.

\begin{tabular}{lll}
\hline Molecule & Theory $^{\mathrm{a}}$ & Expt. $(\AA)$ \\
& $\mathrm{R}_{\mathrm{e}}(\AA)$ & \\
\hline $\mathrm{AgH}$ & 1.614 & $1.6179\left(\mathrm{r}_{\mathrm{e}}\right)^{129}$ \\
$\mathrm{AgF}$ & 1.981 & $1.983179(1)\left(\mathrm{r}_{\mathrm{e}}\right)^{130}$ \\
\hline
\end{tabular}




\begin{tabular}{|c|c|c|}
\hline $\mathrm{AgCl}$ & 2.283 & $2.2807916(6)\left(\mathrm{r}_{\mathrm{e}}\right)^{131}$ \\
\hline $\mathrm{AgBr}$ & 2.400 & $2.393109(2)\left(r_{\mathrm{e}}\right)^{132}$ \\
\hline $\mathrm{AgI}$ & 2.558 & $2.5446165(12)\left(\mathrm{r}_{\mathrm{e}}\right)^{132,133}$ \\
\hline $\mathrm{Ag}_{2}$ & 2.560 & $2.53096(48)\left(\mathrm{r}_{\mathrm{e}}\right)^{134}$ \\
\hline \multicolumn{3}{|l|}{$\mathrm{AgCN}$} \\
\hline$(\mathrm{Ag}-\mathrm{C})$ & 2.015 & $2.0289^{135,136 b}$ \\
\hline$(\mathrm{C}-\mathrm{N})$ & 1.156 & $1.1632^{135,136 b}$ \\
\hline \multicolumn{3}{|l|}{$\mathrm{IAgPH}_{3}$} \\
\hline$(\mathrm{Ag}-\mathrm{I})$ & 2.563 & $2.5483(1)\left(r_{0}\right)^{137}$ \\
\hline$(\mathrm{Ag}-\mathrm{P})$ & 2.341 & $2.3488(20)\left(\mathrm{r}_{0}\right)^{137}$ \\
\hline \multicolumn{3}{|l|}{$\mathrm{IAgNH}_{3}$} \\
\hline$(\mathrm{Ag}-\mathrm{I})$ & 2.549 & $2.5375(3)\left(\mathrm{r}_{0}\right)^{138}$ \\
\hline$(\mathrm{Ag}-\mathrm{N})$ & 2.176 & $2.180(1)\left(r_{0}\right)^{138}$ \\
\hline \multicolumn{3}{|l|}{$\mathrm{IAgCO}$} \\
\hline$(\mathrm{Ag}-\mathrm{I})$ & 2.540 & $2.530(2)\left(r_{s}\right)^{128}$ \\
\hline$(\mathrm{Ag}-\mathrm{C})$ & 2.008 & $2.053(2)\left(\mathrm{r}_{\mathrm{s}}\right)^{128}$ \\
\hline$(\mathrm{C}-\mathrm{O})$ & 1.123 & $1.1236(5)\left(\mathrm{r}_{\mathrm{s}}\right)^{128}$ \\
\hline \multicolumn{3}{|l|}{$\mathrm{ClAgNH}_{3}$} \\
\hline$(\mathrm{Cl}-\mathrm{Ag})$ & 2.267 & $2.2633(9)\left(r_{s}\right)^{139}$ \\
\hline$(\mathrm{Ag}-\mathrm{N})$ & 2.145 & $2.1545(8)\left(r_{s}\right)^{139}$ \\
\hline
\end{tabular}

\footnotetext{
a PBE0/aug-cc-pVTZ, see Section 2.1.1 for details. b Semi-experimental value obtained by combing the ab initio vibration-rotation interaction constants from the composite PESs with the experimental ground state rotational constants, see ref. ${ }^{136}$
}

As the reliability of DLPNO-CCSD(T) based thermochemical approach has been demonstrated in a number of studies, ${ }^{60-63}$ it has been further utilized to examine existing gas phase thermochemical data. Comparison of our predicted gas phase formation enthalpies and absolute 
entropies with corresponding experimental and theoretical values from other groups reported in the literature is summarized in Table 2. Composed chemical equations and corresponding DLPNO$\operatorname{CCSD}(\mathrm{T})$ reaction enthalpies employed to derive the heats of formation, are listed in Table S3. Individual contributions to reaction enthalpies from correlation energy and thermal enthalpy correction are also given in Table S3 to estimate the effect of error compensation in composed reaction schemes. Corresponding $T_{1}$ and $T_{2}$ calculated values are reported in Table S4.

For $\mathrm{AgH}$ the most accurate heat of formation was derived by us from the dissociation energy $\mathrm{D}_{\mathrm{e}}$ given in ref. ${ }^{129}$ and amounts to $64.3 \pm 0.9 \mathrm{kcal} / \mathrm{mol}$. Our predicted value is only $0.3 \mathrm{kcal} / \mathrm{mol}$ higher and is within the experimental uncertainty. The experimental data taken from a compilation of Lias et al. ${ }^{140}$ and used in PM7 parameterization ${ }^{141}$ is $2.6 \mathrm{kcal} / \mathrm{mol}$ higher and apparently less accurate. The most recent experimental heat for the formation of silver fluoride is $4.8 \pm 1.0$ as measured by Hildenbrand et al. ${ }^{142}$ Our value is only $0.2 \mathrm{kcal} / \mathrm{mol}$ higher. The experimental value used in MOPAC reference database ${ }^{141}$ is $2.8 \mathrm{kcal} / \mathrm{mol}$ lower. Similarly, predicted in this work $\Delta \mathrm{H}_{\mathrm{f}}$ of $\mathrm{AgBr}$ is only $0.1 \mathrm{kcal} / \mathrm{mol}$ below the most recent experimental value of $27.8 \pm 0.3 \mathrm{kcal} / \mathrm{mol} .{ }^{143}$ The MOPAC reference value of $25.0 \mathrm{kcal} / \mathrm{mol}$ taken from ref. ${ }^{140}$ is likely less accurate. The predicted AgI $\Delta \mathrm{H}_{\mathrm{f}}$ turned out to be $2.4 \mathrm{kcal} / \mathrm{mol}$ higher than the recent experimental value of $33.4 \pm 1.0$ $\mathrm{kcal} / \mathrm{mol} .{ }^{142}$ As the value tabulated in another compilation ${ }^{28}$ is $38.1 \mathrm{kcal} / \mathrm{mol}$, we expect our $\Delta \mathrm{H}_{\mathrm{f}}$ is reasonable. For $\mathrm{Ag}$ dimer predicted $\Delta \mathrm{H}_{\mathrm{f}}$ turned out to be $1.4 \mathrm{kcal} / \mathrm{mol}$ lower than the experimental value of $98.0 \pm 0.9 \mathrm{kcal} / \mathrm{mol}$ determined by Ran et al. ${ }^{144}$

The experimental formation enthalpies derived from mass-spectroscopic measurements are available for $\mathrm{Ag}_{2} \mathrm{~S}(94.1 \pm 5 \mathrm{kcal} / \mathrm{mol}), \mathrm{Ag}_{2} \mathrm{Se}(83.1 \pm 6 \mathrm{kcal} / \mathrm{mol})$, and $\mathrm{Ag}_{2} \mathrm{Te}(91.3 \pm 5.3 \mathrm{kcal} / \mathrm{mol})$ in a compilation of Krasnov and co-workers. ${ }^{29}$ Remarkably, our $\Delta \mathrm{H}_{\mathrm{f}}$ for these compounds turned out to be very close and amount to $91.2,86.7$ and $90.8 \mathrm{kcal} / \mathrm{mol}$ for $\mathrm{Ag}_{2} \mathrm{~S}, \mathrm{Ag}_{2} \mathrm{Se}$ and $\mathrm{Ag}_{2} \mathrm{Te}$, 
respectively. For $\mathrm{AgCN}$ no experimental heat of formation is available. The best estimate based on $0 \mathrm{~K} \Delta \mathrm{H}_{\mathrm{f}} \mathrm{CCSD}(\mathrm{T})-\mathrm{F} 12$ calculations of Peterson and co-workers ${ }^{136}$ results in $87.9 \mathrm{kcal} / \mathrm{mol}$ which is reasonably close to our value of $86.4 \mathrm{kcal} / \mathrm{mol}$. The deviation of $1.5 \mathrm{kcal} / \mathrm{mol}$ can be explained by more rigorous FPD approach utilized in the work of Peterson and co-workers ${ }^{136}$ where the canonical $\operatorname{CCSD}(\mathrm{T})$ geometries and harmonic frequencies extrapolated to $\mathrm{CBS}$ were used, and the effects arising from ECPs and the full triples have been taken into account. Our predicted $\mathrm{AgPO}_{2} \Delta \mathrm{H}_{\mathrm{f}}$ value of $-63.2 \mathrm{kcal} / \mathrm{mol}$ turned out to be remarkably close to $0 \mathrm{~K} \Delta \mathrm{H}_{\mathrm{f}}$ value of $-60.1 \pm 9.6 \mathrm{kcal} / \mathrm{mol}$ tabulated in the compilation of Krasnov et al. ${ }^{29}$

To estimate an intrinsic error of the composite approach utilized in this work to derive the heats of formation, a few additional calculations have been performed. First, all reaction energies related to the heats of formation listed in Table 2 have been recomputed with the scalar relativistic secondorder Douglas-Kroll-Hess approximation and corresponding relativistically contracted aug-ccpwCVTZ-DK basis sets. ${ }^{145}$ On average, the effect from introducing ECPs and ignoring scalar relativistic effects for the light elements turned out to be $0.3 \mathrm{kcal} / \mathrm{mol}$, see Table S5. Second, all reaction energies have been recalculated with canonical $\operatorname{CCSD}(\mathrm{T})$ and aug-cc-pwCVTZ(-PP) basis sets. As can be seen from Table S5, an average error due to DLPNO approximation amounts to $0.6 \mathrm{kcal} / \mathrm{mol}$. Combination of these results with estimated earlier effects arising from scaling of vibrational frequencies $(0.1 \mathrm{kcal} / \mathrm{mol})$, PBE0 geometries $(0.1 \mathrm{kcal} / \mathrm{mol})$ indicates an overall inaccuracy related to computational scheme utilized in the current work to be within $\pm 2 \mathrm{kcal} / \mathrm{mol}$. Hence, all the heats of formation derived in this work are given with $\pm 2 \mathrm{kcal} / \mathrm{mol}$ uncertainty related to computational protocol plus an uncertainty arising from using the reference heats of formation. An uncertainty of $\pm 1 \mathrm{cal} /(\mathrm{mol} \mathrm{K})$ was ascribed to all entropies calculated in this work. 
Good-to-excellent agreement of the reference data obtained in this work with available experimental measurements indicates the values obtained in our work to be of high accuracy. Hence, a reduced version of the reaction-based FPD protocol can be recommended as a source of reliable thermochemical data.

Table 2. Predicted gas phase heats of formation in $\mathrm{kcal} / \mathrm{mol}$ and absolute entropies in cal $/(\mathrm{mol} \mathrm{K})$ obtained for a few silver species at $298.15 \mathrm{~K}$ for which relevant thermochemical data are reported in the literature.

\begin{tabular}{|c|c|c|c|c|}
\hline \multirow{3}{*}{ Molecule } & $\Delta \mathrm{H}_{\mathrm{f}}(298)$ & $\Delta \mathrm{H}_{\mathrm{f}}(298)$ & $\mathrm{S}^{\circ}(298)$ & $\mathrm{S}^{\circ}(298)$ \\
\hline & {$[\mathrm{kcal} / \mathrm{mol}]$} & {$[\mathrm{kcal} / \mathrm{mol}]$} & {$[\mathrm{cal} /(\mathrm{mol} \mathrm{K})]$} & {$[\mathrm{cal} /(\mathrm{mol} \mathrm{K})]$} \\
\hline & Theory & Expt. & Theory & Expt. \\
\hline \multirow[t]{2}{*}{$\mathrm{AgH}$} & $64.6 \pm 2.3^{\mathrm{a}}$ & $67.0,{ }^{140} 66.3^{28}$ & $48.8 \pm 1.0^{\mathrm{c}}$ & $48.93 \pm 0.02^{146}$ \\
\hline & & $64.3 \pm 0.9^{129, \mathrm{~b}}$ & & \\
\hline $\mathrm{AgF}$ & $5.0 \pm 2.3^{\mathrm{a}}$ & $\begin{array}{l}2.0,{ }^{140} \\
4.8 \pm 1.0^{142}\end{array}$ & $56.3 \pm 1.0^{\mathrm{c}}$ & $56.0 \pm 1.0^{146}$ \\
\hline \multirow[t]{2}{*}{$\mathrm{AgBr}$} & $27.7 \pm 2.4^{\mathrm{a}}$ & $\begin{array}{l}25.0,{ }^{140} \\
23.04 \pm 2.10,{ }^{28}\end{array}$ & $61.4 \pm 1.0^{\mathrm{c}}$ & $61.2 \pm 0.5^{146}$ \\
\hline & & $27.8 \pm 0.3^{143}$ & & \\
\hline \multirow[t]{2}{*}{ AgI } & $35.8 \pm 2.3^{\mathrm{a}}$ & $38.1,,^{28}$ & $63.2 \pm 1.0^{\mathrm{c}}$ & $63.0 \pm 0.5^{146}$ \\
\hline & & $33.4 \pm 1.0^{142}$ & & \\
\hline \multirow[t]{2}{*}{$\mathrm{Ag}_{2}$} & $96.6 \pm 2.6^{\mathrm{a}}$ & $98.0 \pm 0.9^{144}$ & $61.6 \pm 1.0^{\mathrm{c}}$ & $61.39 \pm 0.2^{147}$ \\
\hline & & $97.3^{28}$ & & \\
\hline $\mathrm{Ag}_{2} \mathrm{~S}$ & $91.2 \pm 2.7^{\mathrm{a}}$ & $94.1 \pm 5.0^{29}$ & $76.7 \pm 1.0^{\mathrm{c}}$ & \\
\hline $\mathrm{Ag}_{2} \mathrm{Se}$ & $86.7 \pm 3.3^{\mathrm{a}}$ & $83.1 \pm 6^{29}$ & $79.6 \pm 1.0^{\mathrm{c}}$ & \\
\hline
\end{tabular}



$\mathrm{Ag}_{2} \mathrm{Te} \quad 90.8 \pm 2.8^{\mathrm{a}}$
$91.3 \pm 5.3^{29}$
$81.6 \pm 1.0^{\mathrm{c}}$

$\mathrm{AgCN} \quad 86.4 \pm 2.3^{\mathrm{a}}$

$87.9^{136, \mathrm{~d}}$ (Theor. $62.5 \pm 1.0^{\mathrm{c}}$

FPD)

$\begin{array}{llll}\mathrm{AgPO}_{2} & -63.2 \pm 2.7^{\mathrm{a}} & -60.1 \pm 9.6 & 75.0 \pm 1.0^{\mathrm{c}}\end{array}$

a An uncertainty is calculated as the sum of absolute values of uncertainties of individual reference heats of formation in Table $\mathrm{S} 1$ plus additional $2 \mathrm{kcal} / \mathrm{mol}$ related to applied computational scheme ${ }^{b}$ Derived from dissociation energies $\left(\Delta \mathrm{H}_{\mathrm{f}}(\mathrm{Ag}, 298 \mathrm{~K})=67.1 \pm 0.2\right.$ $\mathrm{kcal} / \mathrm{mol}^{122}, \Delta \mathrm{H}_{\mathrm{f}}(\mathrm{H}, 298 \mathrm{~K})=52.103 \pm 0.000 \mathrm{kcal} / \mathrm{mol}^{119}, \Delta \mathrm{H}_{\text {corr }}($ atom, $298 \mathrm{~K})=1.481 \mathrm{kcal} / \mathrm{mol}$, $\left.\Delta \mathrm{H}_{\text {corr }}(\mathrm{AgH}, 298 \mathrm{~K})=4.591 \mathrm{kcal} / \mathrm{mol}\left(\mathrm{R}_{\mathrm{e}}=1.6179, \omega_{\mathrm{e}}=1759.96 \mathrm{~cm}^{-1}\right)^{129}\right){ }^{\mathrm{c}}$ An uncertainty of \pm 1.0 $\mathrm{cal} /(\mathrm{mol} \mathrm{K})$ has been assigned to all entropies derived in this work based on comparison of scaled and unscaled frequencies derived entropies. ${ }^{\mathrm{d}}$ Estimated from ab initio heat of formation at $0 \mathrm{~K}$ $\left(\Delta \mathrm{H}_{\mathrm{f}}(\mathrm{C}, 298 \mathrm{~K})=171.34 \pm 0.02,{ }^{119} \Delta \mathrm{H}_{\mathrm{f}}(\mathrm{N}, 298 \mathrm{~K})=112.92 \pm 0.01^{119}\right)$

\subsubsection{Data for Silver Compounds with No Experimental Gas Phase Data Available}

Calculated gas phase heats of formation and entropies of 86 silver compounds with no experimental or theoretical data available are listed in Table 3. Corresponding $\mathrm{T}_{1}$ diagnostic values and the largest double excitation amplitudes $\left(\mathrm{T}_{2}\right)$ are reported in Table S6. Composed chemical equations and corresponding DLPNO-CCSD(T) reaction enthalpies employed to derive the heats of formation are listed in Table S3.

Spatial structures of several silver species representing the classes of complexes for which ground state molecular geometries are not trivial are given in Figure 1. PBE0/aug-cc-pVTZ(-PP) Cartesian structures of higher energy conformers/isomers and relative to the ground structures electronic energies are provided in SI. As the higher energy conformers for $\mathrm{Ag}_{2} \mathrm{SO}_{4}, \mathrm{Ag}_{2} \mathrm{SeO}_{4}$ and $\mathrm{Ag}_{2} \mathrm{TeO}_{4}$ turned out to be only a few $\mathrm{kcal} / \mathrm{mol}$ less stable than respective ground state structures, 
these have been also considered and abbreviated further on as $\mathrm{Ag}_{2} \mathrm{SO}_{4}$ (isomer), $\mathrm{Ag}_{2} \mathrm{SeO}_{4}$ (isomer) and $\mathrm{Ag}_{2} \mathrm{TeO}_{4}$ (isomer). 

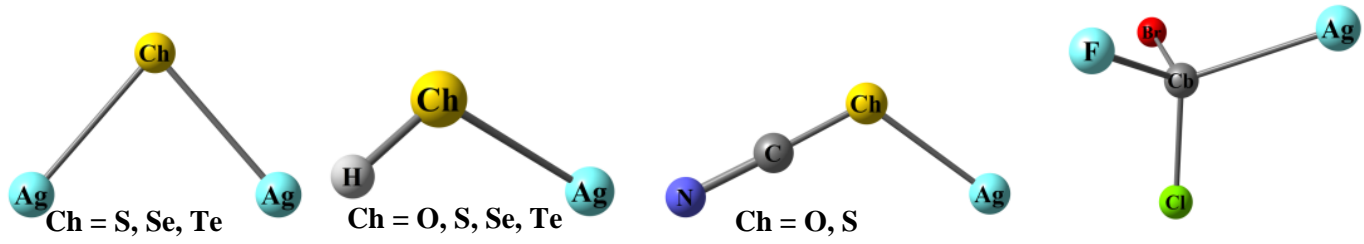

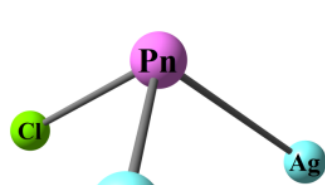

F

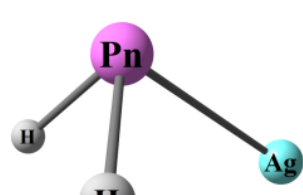

H)
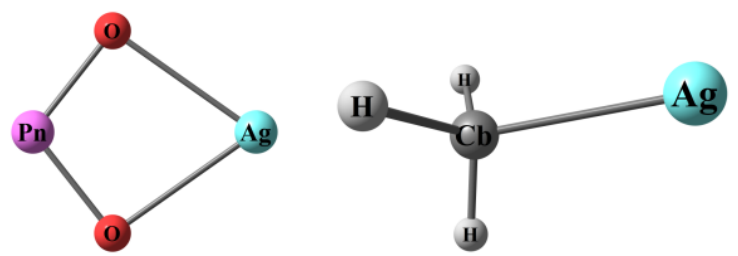

$\mathbf{P n}=\mathbf{N}, \mathbf{P}, \mathbf{A s}, \mathbf{S b}$
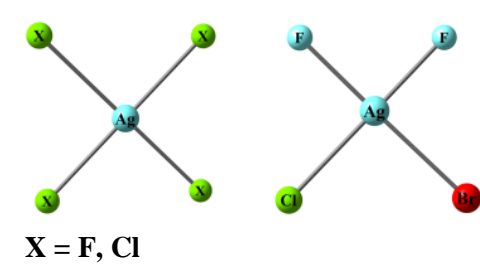

$\mathbf{X}=\mathbf{F}, \mathbf{C l}$

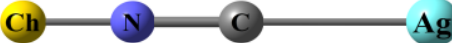

$\mathrm{Ch}=\mathbf{O}, \mathrm{S}$
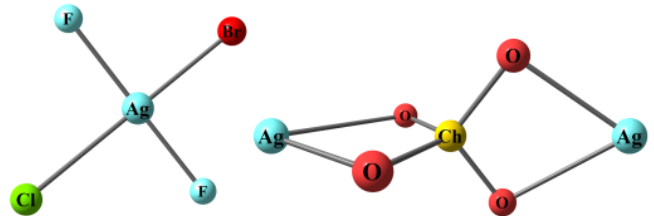

$\mathrm{Cb}=\mathrm{C}, \mathrm{Si}, \mathrm{Ge}, \mathrm{Sn}$

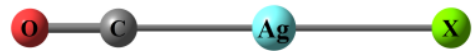

$\mathrm{X}=\mathrm{F}, \mathrm{Cl}, \mathrm{Br}, \mathrm{I}$

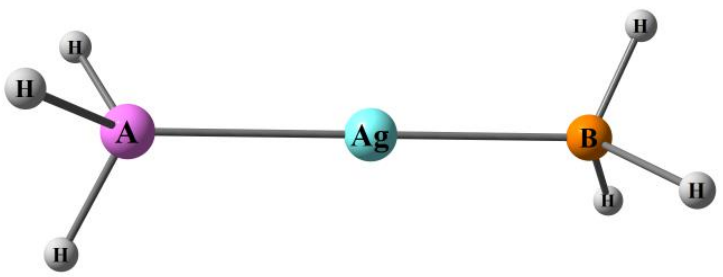

$A=C, N, A s ; B=N, P$
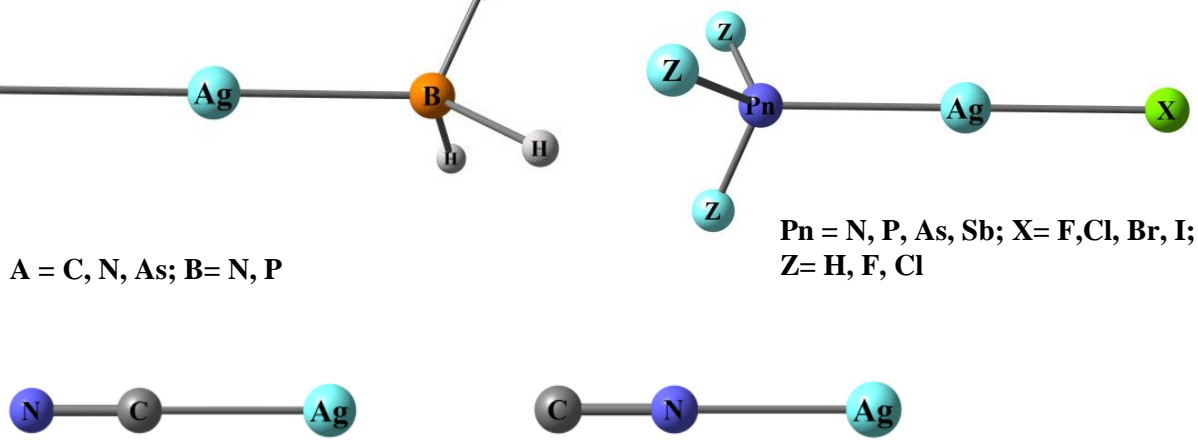

Figure 1. Spatial structures of several silver species for which ground state molecular geometries are not immediately obvious. 
Table 3. DLPNO-CCSD(T)/CBS heats of formation (in $\mathrm{kcal} / \mathrm{mol}$ ) obtained on DFT optimized geometries.

\begin{tabular}{|c|c|c|}
\hline & $\begin{array}{l}\Delta \mathrm{H}_{\mathrm{f}}(298) \\
{[\mathrm{kcal} / \mathrm{mol}]}\end{array}$ & $\begin{array}{l}\mathrm{S}^{\circ}(298) \\
{[\mathrm{cal} /(\mathrm{mol}} \\
\mathrm{K})]\end{array}$ \\
\hline $\mathrm{AgNH}_{2}$ & $68.2 \pm 2.3^{\mathrm{a}}$ & $62.0 \pm 1.0^{\mathrm{b}}$ \\
\hline $\mathrm{AgPH}_{2}$ & $52.5 \pm 2.7^{\mathrm{a}}$ & $66.2 \pm 1.0^{\mathrm{b}}$ \\
\hline $\mathrm{AgAsH}_{2}$ & $60.8 \pm 2.6^{\mathrm{a}}$ & $69.3 \pm 1.0^{\mathrm{b}}$ \\
\hline $\mathrm{AgSbH}_{2}$ & $72.6 \pm 2.4^{\mathrm{a}}$ & $71.7 \pm 1.0^{\mathrm{b}}$ \\
\hline $\mathrm{AgF}_{4}^{-}$ & $-198.9 \pm 2.4^{\mathrm{a}}$ & $76.5 \pm 1.0^{\mathrm{b}}$ \\
\hline $\mathrm{AgCl}_{4}^{-}$ & $-105.7 \pm 2.3^{\mathrm{a}}$ & $92.9 \pm 1.0^{\mathrm{b}}$ \\
\hline $\mathrm{AgBrClF}_{2}^{-}$(cis) & $-144.8 \pm 2.4^{\mathrm{a}}$ & $89.7 \pm 1.0^{\mathrm{b}}$ \\
\hline $\mathrm{AgBrClF}_{2}^{-}$(trans) & $-143.9 \pm 2.4^{\mathrm{a}}$ & $89.9 \pm 1.0^{\mathrm{b}}$ \\
\hline $\mathrm{AgC}_{2} \mathrm{~N}_{2}^{-}$ & $20.3 \pm 2.4^{\mathrm{a}}$ & $75.2 \pm 1.0^{\mathrm{b}}$ \\
\hline $\mathrm{AgTe}^{-}$ & $27.7 \pm 2.5^{\mathrm{a}}$ & $63.2 \pm 1.0^{\mathrm{b}}$ \\
\hline $\mathrm{H}_{3} \mathrm{CAgNH}_{3}$ & $24.6 \pm 2.3^{\mathrm{a}}$ & $72.3 \pm 1.0^{\mathrm{b}}$ \\
\hline $\mathrm{AgCH}_{3}$ & $57.5 \pm 2.4^{\mathrm{a}}$ & $60.7 \pm 1.0^{\mathrm{b}}$ \\
\hline
\end{tabular}




\begin{tabular}{|c|c|c|}
\hline $\mathrm{AgSiH}_{3}$ & $60.0 \pm 2.8^{\mathrm{a}}$ & $65.4 \pm 1.0^{b}$ \\
\hline $\mathrm{AgGeH}_{3}$ & $72.8 \pm 2.5^{\mathrm{a}}$ & $68.4 \pm 1.0^{\mathrm{b}}$ \\
\hline $\mathrm{AgSnH}_{3}$ & $78.1 \pm 3.5^{\mathrm{a}}$ & $71.2 \pm 1.0^{\mathrm{b}}$ \\
\hline $\mathrm{AgNO}_{2}$ & $33.4 \pm 2.4^{\mathrm{a}}$ & $71.9 \pm 1.0^{\mathrm{b}}$ \\
\hline $\mathrm{AgAsO}_{2}$ & $-19.9 \pm 2.6^{\mathrm{a}}$ & $77.6 \pm 1.0^{\mathrm{b}}$ \\
\hline $\mathrm{AgSbO}_{2}$ & $0.4 \pm 2.5^{\mathrm{a}}$ & $79.7 \pm 1.0^{\mathrm{b}}$ \\
\hline $\mathrm{AgOH}$ & $19.3 \pm 2.3^{\mathrm{a}}$ & $60.8 \pm 1.0^{\mathrm{b}}$ \\
\hline $\mathrm{AgSH}$ & $44.5 \pm 2.4^{\mathrm{a}}$ & $64.4 \pm 1.0^{\mathrm{b}}$ \\
\hline $\mathrm{AgSeH}$ & $45.9 \pm 3.0^{\mathrm{a}}$ & $67.6 \pm 1.0^{\mathrm{b}}$ \\
\hline $\mathrm{AgTeH}$ & $58.2 \pm 2.5^{\mathrm{a}}$ & $69.8 \pm 1.0^{\mathrm{b}}$ \\
\hline $\mathrm{AgNC}$ & $99.7 \pm 2.4^{\mathrm{a}}$ & $65.0 \pm 1.0^{\mathrm{b}}$ \\
\hline $\mathrm{AgCNS}$ & $117.4 \pm 2.4^{\mathrm{a}}$ & $73.3 \pm 1.0^{b}$ \\
\hline $\mathrm{AgSCN}$ & $80.3 \pm 2.5^{\mathrm{a}}$ & $75.7 \pm 1.0^{b}$ \\
\hline $\mathrm{AgCNO}$ & $92.5 \pm 2.8^{\mathrm{a}}$ & $67.0 \pm 1.0^{\mathrm{b}}$ \\
\hline $\mathrm{AgOCN}$ & $51.2 \pm 2.4^{\mathrm{a}}$ & $72.1 \pm 1.0^{\mathrm{b}}$ \\
\hline$\left(\mathrm{H}_{3} \mathrm{NAgNH}_{3}\right)^{+}$ & $129.4 \pm 2.4^{\mathrm{a}}$ & $70.9 \pm 1.0^{b}$ \\
\hline
\end{tabular}




\begin{tabular}{|c|c|c|}
\hline$\left(\mathrm{H}_{3} \mathrm{PAgNH}_{3}\right)^{+}$ & $143.2 \pm 2.7^{\mathrm{a}}$ & $76.8 \pm 1.0^{\mathrm{b}}$ \\
\hline$\left(\mathrm{H}_{3} \mathrm{AsAgPH}_{3}\right)^{+}$ & $178.1 \pm 3.0^{\mathrm{a}}$ & $92.1 \pm 1.0^{\mathrm{b}}$ \\
\hline FAgCO & $-50.0 \pm 2.3^{\mathrm{a}}$ & $70.3 \pm 1.0^{\mathrm{b}}$ \\
\hline $\mathrm{ClAgCO}$ & $-28.7 \pm 2.3^{\mathrm{a}}$ & $73.6 \pm 1.0^{\mathrm{b}}$ \\
\hline $\mathrm{BrAgCO}$ & $-21.4 \pm 2.3^{\mathrm{a}}$ & $76.7 \pm 1.0^{\mathrm{b}}$ \\
\hline $\mathrm{IAgCO}$ & $-11.1 \pm 2.3^{\mathrm{a}}$ & $79.1 \pm 1.0^{\mathrm{b}}$ \\
\hline $\mathrm{Ag}_{2} \mathrm{SO}_{4}$ & $-80.2 \pm 2.7^{\mathrm{a}}$ & $97.7 \pm 1.0^{\mathrm{b}}$ \\
\hline $\mathrm{Ag}_{2} \mathrm{SO}_{4}$ (isomer) & $-75.1 \pm 2.7^{\mathrm{a}}$ & $98.1 \pm 1.0^{\mathrm{b}}$ \\
\hline $\mathrm{Ag}_{2} \mathrm{SeO}_{4}$ & $-8.4 \pm 3.4^{\mathrm{a}}$ & $98.0 \pm 1.0^{\mathrm{b}}$ \\
\hline $\mathrm{Ag}_{2} \mathrm{SeO}_{4}$ (isomer) & $-3.1 \pm 3.4^{\mathrm{a}}$ & $101.3 \pm 1.0^{\mathrm{b}}$ \\
\hline $\mathrm{Ag}_{2} \mathrm{TeO}_{4}$ & $-2.2 \pm 2.8^{\mathrm{a}}$ & $101.8 \pm 1.0^{\mathrm{b}}$ \\
\hline $\mathrm{Ag}_{2} \mathrm{TeO}_{4}$ (isomer) & $2.6 \pm 2.8^{\mathrm{a}}$ & $104.7 \pm 1.0^{\mathrm{b}}$ \\
\hline $\mathrm{FAgPH}_{3}$ & $-29.2 \pm 2.8^{\mathrm{a}}$ & $76.1 \pm 1.0^{\mathrm{b}}$ \\
\hline $\mathrm{ClAgPH}_{3}$ & $-8.7 \pm 2.7^{\mathrm{a}}$ & $79.4 \pm 1.0^{\mathrm{b}}$ \\
\hline $\mathrm{BrAgPH}_{3}$ & $-1.5 \pm 2.7^{\mathrm{a}}$ & $82.4 \pm 1.0^{\mathrm{b}}$ \\
\hline $\mathrm{IAgPH}_{3}$ & $8.6 \pm 2.7^{\mathrm{a}}$ & $84.6 \pm 1.0^{\mathrm{b}}$ \\
\hline
\end{tabular}




\begin{tabular}{|c|c|c|}
\hline $\mathrm{BrAgPCl}_{3}$ & $-64.2 \pm 3.6^{\mathrm{a}}$ & $105.7 \pm 1.0^{\mathrm{b}}$ \\
\hline $\mathrm{BrAgPF}_{3}$ & $-225.7 \pm 3.2^{\mathrm{a}}$ & $96.1 \pm 1.0^{b}$ \\
\hline $\mathrm{FAgPF}_{3}$ & $-254.6 \pm 3.2^{\mathrm{a}}$ & $89.6 \pm 1.0^{b}$ \\
\hline $\mathrm{FAgPCl}_{3}$ & $-92.1 \pm 3.6^{\mathrm{a}}$ & $99.2 \pm 1.0^{\mathrm{b}}$ \\
\hline $\mathrm{ClAgPCl}_{3}$ & $-71.3 \pm 3.6^{\mathrm{a}}$ & $102.6 \pm 1.0^{\mathrm{b}}$ \\
\hline $\mathrm{ClAgPF}_{3}$ & $-233.1 \pm 3.3^{\mathrm{a}}$ & $93.0 \pm 1.0^{\mathrm{b}}$ \\
\hline $\mathrm{IAgPCl}_{3}$ & $-54.1 \pm 3.6^{\mathrm{a}}$ & $108.1 \pm 1.0^{\mathrm{b}}$ \\
\hline $\mathrm{IAgPF}_{3}$ & $-215.2 \pm 3.3^{\mathrm{a}}$ & $98.4 \pm 1.0^{\mathrm{b}}$ \\
\hline $\mathrm{FAgAsH}_{3}$ & $-9.1 \pm 2.6^{\mathrm{a}}$ & $79.7 \pm 1.0^{\mathrm{b}}$ \\
\hline $\mathrm{ClAgAsH}_{3}$ & $10.6 \pm 2.6^{\mathrm{a}}$ & $83.0 \pm 1.0^{b}$ \\
\hline $\mathrm{BrAgAsH}_{3}$ & $17.6 \pm 2.6^{\mathrm{a}}$ & $86.0 \pm 1.0^{\mathrm{b}}$ \\
\hline $\mathrm{IAgAsH}_{3}$ & $27.4 \pm 2.6^{\mathrm{a}}$ & $88.2 \pm 1.0^{\mathrm{b}}$ \\
\hline $\mathrm{FAgSbH}_{3}$ & $10.0 \pm 2.4^{\mathrm{a}}$ & $82.7 \pm 1.0^{\mathrm{b}}$ \\
\hline $\mathrm{ClAgSbH}_{3}$ & $29.6 \pm 2.4^{\mathrm{a}}$ & $85.9 \pm 1.0^{\mathrm{b}}$ \\
\hline $\mathrm{BrAgSbH}_{3}$ & $36.6 \pm 2.4^{\mathrm{a}}$ & $88.9 \pm 1.0^{b}$ \\
\hline $\mathrm{IAgSbH}_{3}$ & $46.4 \pm 2.4^{\mathrm{a}}$ & $91.1 \pm 1.0^{\mathrm{b}}$ \\
\hline
\end{tabular}




\begin{tabular}{|c|c|c|}
\hline $\mathrm{FAgNH}_{3}$ & $-38.1 \pm 2.3^{a}$ & $72.2 \pm 1.0^{\mathrm{b}}$ \\
\hline $\mathrm{ClAgNH}_{3}$ & $-19.6 \pm 2.4^{\mathrm{a}}$ & $75.3 \pm 1.0^{\mathrm{b}}$ \\
\hline $\mathrm{BrAgNH}_{3}$ & $-12.8 \pm 2.4^{\mathrm{a}}$ & $78.2 \pm 1.0^{\mathrm{b}}$ \\
\hline $\mathrm{IAgNH}_{3}$ & $-3.3 \pm 2.3^{\mathrm{a}}$ & $80.3 \pm 1.0^{\mathrm{b}}$ \\
\hline $\mathrm{FAgNF}_{3}$ & $-37.1 \pm 2.6^{\mathrm{a}}$ & $87.8 \pm 1.0^{b}$ \\
\hline $\mathrm{BrAgNF}_{3}$ & $-12.1 \pm 2.6^{\mathrm{a}}$ & $94.8 \pm 1.0^{b}$ \\
\hline $\mathrm{ClAgNF}_{3}$ & $-18.6 \pm 2.6^{\mathrm{a}}$ & $91.6 \pm 1.0^{\mathrm{b}}$ \\
\hline $\mathrm{IAgNF}_{3}$ & $-3.0 \pm 2.6^{\mathrm{a}}$ & $97.3 \pm 1.0^{\mathrm{b}}$ \\
\hline $\mathrm{AgCBrClF}$ & $18.9 \pm 2.7^{\mathrm{a}}$ & $86.3 \pm 1.0^{\mathrm{b}}$ \\
\hline $\mathrm{AgSiBrClF}$ & $-104.6 \pm 2.9^{\mathrm{a}}$ & $91.5 \pm 1.0^{b}$ \\
\hline $\mathrm{AgGeBrClF}$ & $-71.1 \pm 2.5^{\mathrm{a}}$ & $96.0 \pm 1.0^{\mathrm{b}}$ \\
\hline $\mathrm{AgSnBrClF}$ & $-77.1 \pm 3.5^{\mathrm{a}}$ & $100.0 \pm 1.0^{\mathrm{b}}$ \\
\hline $\mathrm{AgNClF}$ & $83.2 \pm 2.6^{\mathrm{a}}$ & $76.8 \pm 1.0^{b}$ \\
\hline $\mathrm{AgPClF}$ & $-31.5 \pm 2.8^{\mathrm{a}}$ & $80.0 \pm 1.0^{\mathrm{b}}$ \\
\hline $\mathrm{AgAsClF}$ & $-24.3 \pm 2.6^{\mathrm{a}}$ & $83.5 \pm 1.0^{\mathrm{b}}$ \\
\hline $\mathrm{AgSbClF}$ & $-31.0 \pm 2.4^{\mathrm{a}}$ & $86.3 \pm 1.0^{\mathrm{b}}$ \\
\hline
\end{tabular}




\begin{tabular}{|c|c|c|}
\hline $\mathrm{AgHe}^{+}$ & $239.6 \pm 2.3^{\mathrm{a}}$ & $55.4 \pm 1.0^{\natural}$ \\
\hline $\mathrm{AgNe}^{+}$ & $239.1 \pm 2.3^{\mathrm{a}}$ & $60.4 \pm 1.0^{\mathrm{b}}$ \\
\hline $\mathrm{AgAr}^{+}$ & $233.2 \pm 2.3^{\mathrm{a}}$ & $61.2 \pm 1.0^{\mathrm{b}}$ \\
\hline $\mathrm{AgKr}^{+}$ & $229.4 \pm 2.3^{a}$ & $63.1 \pm 1.0^{\mathrm{b}}$ \\
\hline $\mathrm{AgXe}^{+}$ & $223.6 \pm 2.3^{\mathrm{a}}$ & $64.4 \pm 1.0^{\mathrm{b}}$ \\
\hline $\mathrm{AgRn}^{+}$ & $220.8 \pm 2.3^{a}$ & $66.0 \pm 1.0^{\mathrm{b}}$ \\
\hline $\mathrm{AgBe}^{+}$ & $278.0 \pm 2.3^{\mathrm{a}}$ & $55.3 \pm 1.0^{\mathrm{b}}$ \\
\hline $\mathrm{AgCa}^{+}$ & $208.7 \pm 2.5^{\mathrm{a}}$ & $60.8 \pm 1.0^{\mathrm{b}}$ \\
\hline $\mathrm{AgMg}^{+}$ & $225.4 \pm 2.5^{\mathrm{a}}$ & $58.9 \pm 1.0^{\mathrm{b}}$ \\
\hline $\mathrm{AgSr}^{+}$ & $198.3 \pm 2.7^{\mathrm{a}}$ & $63.4 \pm 1.0^{\mathrm{b}}$ \\
\hline $\mathrm{AgBa}^{+}$ & $187.3 \pm 3.5^{\mathrm{a}}$ & $64.9 \pm 1.0^{\mathrm{b}}$ \\
\hline $\mathrm{AgZn}^{+}$ & $238.2 \pm 2.4^{\mathrm{a}}$ & $61.5 \pm 1.0^{\mathrm{b}}$ \\
\hline $\mathrm{AgCd}^{+}$ & $232.0 \pm 2.4^{\mathrm{a}}$ & $63.5 \pm 1.0^{\mathrm{b}}$ \\
\hline $\mathrm{AgHg}^{+}$ & $229.5 \pm 2.3^{\mathrm{a}}$ & $65.3 \pm 1.0^{\mathrm{h}}$ \\
\hline
\end{tabular}

\footnotetext{
${ }^{a}$ An uncertainty is calculated as the sum of absolute values of uncertainties of individual reference heats of formation in Table $\mathrm{S} 1$ plus additional $2 \mathrm{kcal} / \mathrm{mol}$ related to applied computational scheme. ${ }^{\mathrm{b}}$ An uncertainty of $\pm 1.0 \mathrm{cal} /(\mathrm{mol} \mathrm{K})$ has been assigned to all entropies derived in this work based on a comparison of scaled and unscaled frequencies derived entropies.
} 
The lowest calculated $\Delta \mathrm{H}_{\mathrm{f}}=-254.6 \pm 3.2 \mathrm{kcal} / \mathrm{mol}$ of $\mathrm{FAgPF}_{3}$ and highest $\Delta \mathrm{H}_{\mathrm{f}}=278.0 \pm 2.3$ $\mathrm{kcal} / \mathrm{mol}$ of $\mathrm{AgBe}^{+}$provide a span of $532.6 \mathrm{kcal} / \mathrm{mol}$ which is sufficiently large to both fit and test semiempirical methods. These data would substantially enrich the MOPAC reference data compilation as only two species from Table 3, namely $\mathrm{AgOH}$ and $\mathrm{AgSH}$, are mentioned in there. For silver hydroxide, $\mathrm{AgOH}$, our calculations yielded gas phase $\Delta \mathrm{H}_{\mathrm{f}}$ of $19.3 \pm 2.3 \mathrm{kcal} / \mathrm{mol}$ that is $6.6 \mathrm{kcal} / \mathrm{mol}$ lower than its DFT estimated counterpart utilized to train semiempirical methods in MOPAC. Similarly, the substantial difference has been identified between predicted $\left(\Delta \mathrm{H}_{\mathrm{f}}=\right.$ $44.5 \pm 2.4 \mathrm{kcal} / \mathrm{mol})$ and PM6 reference $\left(\Delta \mathrm{H}_{\mathrm{f}}=40.8 \mathrm{kcal} / \mathrm{mol}\right)$ heats of formation of AgSH. We expect that more accurate $\Delta \mathrm{H}_{\mathrm{f}} \mathrm{AgOH} / \mathrm{AgSH}$ values given in this work together with the data for other 84 species would result in more robust PM6/PM7 parameterization of silver. Based on gas phase formation enthalpy, AgSCN with $\Delta \mathrm{H}_{\mathrm{f}}=80.3 \pm 2.5 \mathrm{kcal} / \mathrm{mol}$ is more stable than AgCNS with $\Delta \mathrm{H}_{\mathrm{f}}=117.4 \pm 2.4 \mathrm{kcal} / \mathrm{mol}$. Gas phase $\mathrm{AgOCN}$ with $\Delta \mathrm{H}_{\mathrm{f}}=51.2 \pm 2.4 \mathrm{kcal} / \mathrm{mol}$ turned out to be more stable than $\mathrm{AgCNO}$ with $\Delta \mathrm{H}_{\mathrm{f}}=92.5 \pm 2.8 \mathrm{kcal} / \mathrm{mol} . \mathrm{AgBrClF}_{2}^{-}$(cis) with $\mathrm{H}_{\mathrm{f}}=-144.8 \pm 2.4 \mathrm{kcal} / \mathrm{mol}$ is more stable than $\mathrm{AgBrClF}_{2}^{-}$(trans) with $\mathrm{H}_{\mathrm{f}}=-143.9 \pm 2.4 \mathrm{kcal} / \mathrm{mol}$. The predicted formation enthalpies $\Delta \mathrm{H}_{\mathrm{f}}$ of $\mathrm{Ag}_{2} \mathrm{SO}_{4}, \mathrm{Ag}_{2} \mathrm{SeO}_{4}$ and $\mathrm{Ag}_{2} \mathrm{TeO}_{4}$ ground state structures (left-hand side geometries in Figure 1) amount to $-80.2 \pm 2.7,-8.4 \pm 3.4$ and $-2.2 \pm 2.8 \mathrm{kcal} / \mathrm{mol}$, correspondingly. The heats of formation of less stable energy isomers turned out to be $5.1,5.3$ and $4.8 \mathrm{kcal} / \mathrm{mol}$ higher for $\mathrm{Ag}_{2} \mathrm{SO}_{4}, \mathrm{Ag}_{2} \mathrm{SeO}_{4}$, and $\mathrm{Ag}_{2} \mathrm{TeO}_{4}$, respectively.

Most of the predicted heats of formations have been further utilized to calculate sublimation thermodynamic data (Section 3.2.1) and bond strengths (Section 3.2.2) of some silver complexes for which relevant experimental data are available.

\subsection{Derived Thermodynamic Quantities}




\subsubsection{Sublimation Thermochemistry}

Combining experimental solid state heats of formation and entropies available for $\mathrm{AgNO}_{2}$, $\mathrm{AgSCN}, \mathrm{Ag}_{2} \mathrm{SO}_{4}$, and $\mathrm{Ag}_{2} \mathrm{SeO}_{4}$ with their gas phase counterparts obtained in this work, we have derived respective thermodynamic functions of sublimation listed in Table 4. In all cases we assumed the saturated vapor over the solids consists only of molecular species $\left(\mathrm{AgNO}_{2}, \mathrm{AgSCN}\right.$, $\left.\mathrm{Ag}_{2} \mathrm{SO}_{4}, \mathrm{Ag}_{2} \mathrm{SeO}_{4}\right)$, i.e. possibility of dimer or trimer forms was not considered. $\Delta \mathrm{H}_{\text {sub }}$ of $\mathrm{AgNO}_{2}$ amounts to $44.2 \pm 2.6 \mathrm{kcal} / \mathrm{mol}$ which turned out to be close to that of $\mathrm{NaNO}_{2}\left(\Delta \mathrm{H}_{\text {sub }}=42.0 \pm 1.0\right),{ }^{28}$ $\mathrm{KNO}_{2}\left(\Delta \mathrm{H}_{\text {sub }}=42.8 \pm 0.8\right)^{28}$ and $\mathrm{RbNO}_{2}\left(\Delta \mathrm{H}_{\text {sub }}=42.2 \pm 1.0\right)^{28}$ indicating similar magnitude/strength of crystal packing forces in these systems. Predicted sublimation entropy change amounts to $\Delta \mathrm{S}_{\text {sub }}$ $=41.3 \pm 1.1 \mathrm{cal} /(\mathrm{mol} \mathrm{K}) . \Delta \mathrm{H}_{\text {sub }}$ of $59.8 \pm 2.5 \mathrm{kcal} / \mathrm{mol}$ of $\mathrm{AgSCN}$ indicates quite large magnitude of crystal packing forces in the crystal. AgSCN sublimation entropy change amounts to $\Delta \mathrm{S}_{\text {sub }}=$ $43.9 \pm 1.1 \mathrm{cal} /(\mathrm{mol} \mathrm{K}) . \mathrm{Ag}_{2} \mathrm{SO}_{4} \Delta \mathrm{H}_{\text {sub }}$ and $\Delta \mathrm{S}_{\text {sub }}$ amount to $91.4 \pm 2.9 \mathrm{kcal} / \mathrm{mol}$ and $49.9 \pm 1.1 \mathrm{cal} /(\mathrm{mol}$ $\mathrm{K})$, correspondingly. Sublimation enthalpy of similar magnitude $\Delta \mathrm{H}_{\text {sub }}=92.6 \pm 3.4 \mathrm{kcal} / \mathrm{mol}$ is obtained for $\mathrm{Ag}_{2} \mathrm{SeO}_{4}$. $\mathrm{Ag}_{2} \mathrm{SeO}_{4} \Delta \mathrm{S}_{\text {sub }}$ was found to be $34.7 \pm 1.1 \mathrm{cal} /(\mathrm{mol} \mathrm{K})$.

Table 4. Solid-state and sublimation thermodynamic functions of a few silver compounds assuming the saturated vapor over the solids consists only of molecular species $\left(\mathrm{AgNO}_{2}, \mathrm{AgSCN}\right.$, $\left.\mathrm{Ag}_{2} \mathrm{SO}_{4}, \mathrm{Ag}_{2} \mathrm{SeO}_{4}\right)$.

\begin{tabular}{lllll}
\hline & $\Delta \mathrm{H}_{\mathrm{f}}(298)$ & $\mathrm{S}^{\circ}(298)$ & $\Delta \mathrm{H}_{\text {sub }}(298)$ & $\Delta \mathrm{S}_{\text {sub }}^{\circ}(298)$ \\
& {$[\mathrm{kcal} / \mathrm{mol}]$} & & {$[\mathrm{kcal} / \mathrm{mol}]$} & \\
& & {$[\mathrm{cal} /(\mathrm{mol} \mathrm{K})]$} & & {$[\mathrm{cal} /(\mathrm{mol} \mathrm{K})]$} \\
& & & & \\
\hline $\mathrm{AgNO}_{2}$ & $-10.8 \pm 0.2^{28}$ & $30.6 \pm 0.1^{28}$ & $44.2 \pm 2.6^{\mathrm{a}}$ & $41.3 \pm 1.1$ \\
& & & & \\
\hline
\end{tabular}




\begin{tabular}{lllll}
\hline $\mathrm{AgSCN}$ & $20.5^{28}$ & $31.8^{28}$ & $59.8 \pm 2.5^{\mathrm{a}}$ & $43.9 \pm 1.1$ \\
$\mathrm{Ag}_{2} \mathrm{SO}_{4}$ & $-171.42 \pm 0.12^{28}$ & $47.76 \pm 0.1^{28}$ & $91.4 \pm 2.9^{\mathrm{a}}$ & $49.9 \pm 1.1$ \\
$\mathrm{Ag}_{2} \mathrm{SeO}_{4}$ & $-100.95^{28}$ & $63.29^{28}$ & $92.6 \pm 3.4^{\mathrm{a}}$ & $34.7 \pm 1.1$
\end{tabular}

\footnotetext{
${ }^{a}$ An uncertainty is calculated as the sum of absolute values of uncertainties of gas phase formation enthalpies in Table 2 and corresponding heats of formation of the solid state species, see main text.
}

\subsubsection{Silver Bond Strengths}

Using additional molecular/atomic reference experimental gas phase formation enthalpies from Tables S1 and S7, we derived a set of bond dissociation enthalpies of 33 silver species listed in Table 5. Comparing to direct calculations via (DLPNO-)CCSD(T) approach implying calculation of atomization-like energies and no error cancellations, we expect our bond strengths are more reliable. The weakest silver bond strength $\Delta \mathrm{H}_{\mathrm{r}}=3.2 \pm 2.5 \mathrm{kcal} / \mathrm{mol}$ has been obtained for $\mathrm{AgHe}^{+} \rightarrow \mathrm{Ag}^{+}+\mathrm{He}$ dissociation. The strongest bond strength $\Delta \mathrm{H}_{\mathrm{r}}=111.2 \pm 3.2 \mathrm{kcal} / \mathrm{mol}$ has been obtained for $\mathrm{Ag}_{2} \mathrm{~S} \rightarrow 2 \mathrm{Ag}+\mathrm{S}$ resulting in an overall span in calculated enthalpies of $108 \mathrm{kcal} / \mathrm{mol}$. The detailed analysis of all the bond strengths obtained in this work is given below.

The strongest $\mathrm{Ag}-\mathrm{Hal}(\mathrm{Hal}=\mathrm{F}, \mathrm{Cl}, \mathrm{Br}, \mathrm{I})$ bond dissociation enthalpy $\Delta \mathrm{H}_{\mathrm{r}}=82.1 \pm 2.5$ $\mathrm{kcal} / \mathrm{mol}$ was calculated for $\mathrm{AgF}$, followed by $\mathrm{AgCl}$ with $\Delta \mathrm{H}_{\mathrm{r}}=75.1 \pm 0.5 \mathrm{kcal} / \mathrm{mol}, \mathrm{AgBr}$ with $\Delta \mathrm{H}_{\mathrm{r}}$ $=67.1 \pm 2.6 \mathrm{kcal} / \mathrm{mol}$ and $\mathrm{AgI}$ with $\Delta \mathrm{H}_{\mathrm{r}}=57.8 \pm 2.5 \mathrm{kcal} / \mathrm{mol} . \mathrm{Ag}-\mathrm{H}$ bond dissociation enthalpy turned out to be only a few $\mathrm{kcal} / \mathrm{mol}$ smaller comparing to that in $\mathrm{Ag}-\mathrm{I}$ and amounts to $\Delta \mathrm{H}_{\mathrm{r}}=$ $55.6 \pm 2.5 \mathrm{kcal} / \mathrm{mol}$. Significantly lower dissociation enthalpy of $\Delta \mathrm{H}_{\mathrm{r}}=39.6 \pm 3.0 \mathrm{kcal} / \mathrm{mol}$ was 
predicted for bond rupture in the silver dimer. The atomization enthalpies for ionic $\mathrm{Ag}_{2} \mathrm{~S}, \mathrm{Ag}_{2} \mathrm{Se}$, and $\mathrm{Ag}_{2} \mathrm{Te}$ molecules were calculated to be $\Delta \mathrm{H}_{\mathrm{r}}=111.2 \pm 3.2 \mathrm{kcal} / \mathrm{mol}, 107.6 \pm 4.0 \mathrm{kcal} / \mathrm{mol}$ and $\approx 96.9 \pm 3.2 \mathrm{kcal} / \mathrm{mol}$, respectively, which is supported by the electronegativity decrease in row $\mathrm{S}-$ $\mathrm{Se}-\mathrm{Te} .{ }^{148}$ The predicted enthalpy change for $\mathrm{Ag}-\mathrm{NH}_{2}$ bond breaking amounts to $44.4 \pm 2.5$ $\mathrm{kcal} / \mathrm{mol}$ which is similar to that of $\mathrm{Ag}-\mathrm{AsH}_{2}$ with $\Delta \mathrm{H}_{\mathrm{r}} \approx 45.3 \pm 2.8 \mathrm{kcal} / \mathrm{mol}$. Remarkably, $\mathrm{AgPH}_{2}$ $\rightarrow \mathrm{Ag}+\mathrm{PH}_{2}$ dissociation with $\Delta \mathrm{H}_{\mathrm{r}} \approx 55.2 \pm 2.9 \mathrm{kcal} / \mathrm{mol}$ turned out to be more energy consuming. Bond strength $\mathrm{Ag}-\mathrm{CH}_{3}$ with $\Delta \mathrm{H}_{\mathrm{r}}=45.6 \pm 2.6 \mathrm{kcal} / \mathrm{mol}$ was predicted to be by $12.9 \mathrm{kcal} / \mathrm{mol}$ weaker than in $\mathrm{AgSiH}_{3}$ with $\Delta \mathrm{H}_{\mathrm{r}}$ of $\approx 58.5 \pm 3.0 \mathrm{kcal} / \mathrm{mol}$ and by $6.7 \mathrm{kcal} / \mathrm{mol}$ weaker than in $\mathrm{AgGeH}_{3}$ with $\Delta \mathrm{H}_{\mathrm{r}}$ of $\approx 52.3 \pm 7.7 \mathrm{kcal} / \mathrm{mol}$. Noteworthy, as reference heats of formation of $\mathrm{SiH}_{3}$ and $\mathrm{GeH}_{3}$ are poorly defined compared to that of $\mathrm{CH}_{3}$, predicted bond dissociation enthalpies of $\mathrm{AgSiH}_{3}$ and $\mathrm{AgGeH}_{3}$ should be taken with caution. $\mathrm{Ag}-\mathrm{OH}$ and $\mathrm{Ag}-\mathrm{SH}$ dissociation enthalpies turned out to be very similar and amount to $\Delta \mathrm{H}_{\mathrm{r}}=57.8 \pm 2.5$ and $58.1 \pm 4.6 \mathrm{kcal} / \mathrm{mol}$, correspondingly.

Finally, a few bond strengths have been calculated for a set of positively charged silver species. First, we derived silver cation - noble gas bond strengths. The weakest dissociation enthalpy $\Delta \mathrm{H}_{\mathrm{r}}=3.2 \pm 2.5 \mathrm{kcal} / \mathrm{mol}$ was predicted for $\mathrm{AgHe}^{+}$, followed by $\mathrm{AgNe}^{+}$with $\Delta \mathrm{H}_{\mathrm{r}}=3.7 \pm 2.5$ $\mathrm{kcal} / \mathrm{mol}$, then by $\mathrm{AgAr}^{+}$with $\Delta \mathrm{H}_{\mathrm{r}}=9.6 \pm 2.5 \mathrm{kcal} / \mathrm{mol}$, then by $\mathrm{AgKr}^{+}$with $\Delta \mathrm{H}_{\mathrm{r}}=13.4 \pm 2.5$ $\mathrm{kcal} / \mathrm{mol}$, then by $\mathrm{AgXe}{ }^{+}$with $\Delta \mathrm{H}_{\mathrm{r}}=19.2 \pm 2.5 \mathrm{kcal} / \mathrm{mol}$, and then by $\mathrm{AgRn}^{+}$with $\Delta \mathrm{H}_{\mathrm{r}}=22.0 \pm 2.5$ $\mathrm{kcal} / \mathrm{mol}$. These values correlate well with decrease of ionization potential (IP) in series He (IP $=567.0 \pm 0.0 \mathrm{kcal} / \mathrm{mol}), \mathrm{Ne}(\mathrm{IP}(\mathrm{Ne})=497.3 \pm 0.0 \mathrm{kcal} / \mathrm{mol}), \mathrm{Ar}(\mathrm{IP}(\mathrm{Ar})=363.4 \pm 0.0 \mathrm{kcal} / \mathrm{mol}), \mathrm{Kr}$ $(\mathrm{IP}(\mathrm{Kr})=322.8 \pm 0.0 \mathrm{kcal} / \mathrm{mol}), \mathrm{Xe}(\mathrm{IP}(\mathrm{Xe})=279.7 \pm 0.0 \mathrm{kcal} / \mathrm{mol})$ and $\mathrm{Rn}(\mathrm{IP}(\mathrm{Rn})=247.9 \pm 0.0$ $\mathrm{kcal} / \mathrm{mol})^{25}$ Second, we analyzed silver cation $-\mathrm{Mg} / \mathrm{Be}$ bond strengths. $\mathrm{Mg}$ atom was found to 
bind stronger by $10.4 \mathrm{kcal} / \mathrm{mol}$ to silver cation than Be atom with $\Delta \mathrm{H}_{\mathrm{r}}($ diss. $)=42.2 \pm 3.7$. Once again, found bond strengths correlate well with a decrease of IP when going from $\mathrm{Be}(\mathrm{IP}(\mathrm{Be})=$ $215.0 \pm 0.0 \mathrm{kcal} / \mathrm{mol})$ to $\mathrm{Mg}(\mathrm{IP}(\mathrm{Mg})=176.4 \pm 0.0 \mathrm{kcal} / \mathrm{mol})$. As ionization potential of $\mathrm{Ag}$ $\left(\mathrm{IP}=174.7123 \pm 0.0003 \mathrm{kcal} / \mathrm{mol}^{25}\right)$ turned out to be larger than that of $\mathrm{Ca}(\mathrm{IP}=141.0 \pm 0.0 \mathrm{kcal} / \mathrm{mol})$ and $\mathrm{Sr}(\mathrm{IP}=131.3 \pm 0.0 \mathrm{kcal} / \mathrm{mol})$, we estimated binding of $\mathrm{Ca}$ and $\mathrm{Sr}$ cations to silver atoms. As expected from higher electron affinity (EA) of calcium ion, it was found to bind stronger to $\mathrm{Ag}$ $\left(\Delta \mathrm{H}_{\mathrm{r}}(\right.$ diss. $\left.)=44.4 \pm 2.7 \mathrm{kcal} / \mathrm{mol}\right)$ than strontium ion $\left(\Delta \mathrm{H}_{\mathrm{r}}(\right.$ diss. $\left.)=41.0 \pm 2.9 \mathrm{kcal} / \mathrm{mol}\right)$. Finally, for silver cation - group 11 atom compounds, the largest dissociation enthalpy $\Delta \mathrm{H}_{\mathrm{r}}=37.5 \pm 2.7$ $\mathrm{kcal} / \mathrm{mol}$ was predicted for $\mathrm{AgCd}^{+}$, followed by $\mathrm{AgZn}^{+}$with $\Delta \mathrm{H}_{\mathrm{r}}=35.8 \pm 2.7 \mathrm{kcal} / \mathrm{mol}$ and then by $\mathrm{AgHg}^{+}$with $\Delta \mathrm{H}_{\mathrm{r}}=28.0 \pm 2.5 \mathrm{kcal} / \mathrm{mol}$. It agrees well with an increase of ionization potential in row $\mathrm{Cd}(\mathrm{IP}=207.4 \pm 0.0 \mathrm{kcal} / \mathrm{mol}), \mathrm{Zn}(\mathrm{IP}=216.6 \pm 0.0 \mathrm{kcal} / \mathrm{mol}), \mathrm{Hg}(\mathrm{IP}=240.7 \pm 0.0 \mathrm{kcal} / \mathrm{mol}) .{ }^{25}$

We expect that derived bond strengths, as well as formation enthalpies, will enrich thermochemistry of reference inorganic silver compounds and contribute further to understanding the chemistry of silver. Apart from that, obtained data are highly relevant to parameterization and benchmarking of semiempirical quantum chemistry methods. Combination of gas phase data with the available data in solution can be used to derive silver compounds solvation thermochemistry database to developed and test explicit and implicit solvation models. ${ }^{149-151}$

Table 5. Gas phase homolytic bond strengths in silver complexes

\begin{tabular}{lc}
\hline Reaction & $\Delta \mathrm{H}_{\mathrm{r}}(298)$ \\
& {$[\mathrm{kcal} / \mathrm{mol}]$} \\
$\mathrm{AgH} \rightarrow \mathrm{Ag}+\mathrm{H}$ & $55.6 \pm 2.5^{\mathrm{a}}$ \\
\hline
\end{tabular}




\begin{tabular}{|c|c|}
\hline $\mathrm{AgF} \rightarrow \mathrm{Ag}+\mathrm{F}$ & $82.1 \pm 2.5^{\mathrm{a}}$ \\
\hline $\mathrm{AgCl} \rightarrow \mathrm{Ag}+\mathrm{Cl}$ & $75.1 \pm 0.5^{\mathrm{b}}$ \\
\hline $\mathrm{AgBr} \rightarrow \mathrm{Ag}+\mathrm{Br}$ & $67.1 \pm 2.6^{\mathrm{a}}$ \\
\hline $\mathrm{AgI} \rightarrow \mathrm{Ag}+\mathrm{I}$ & $57.8 \pm 2.5^{\mathrm{a}}$ \\
\hline $\mathrm{Ag}_{2} \rightarrow 2 \mathrm{Ag}$ & $39.6 \pm 3.0^{\mathrm{a}}$ \\
\hline $\mathrm{Ag}_{2} \mathrm{~S} \rightarrow 2 \mathrm{Ag}+\mathrm{S}$ & $111.2 \pm 3.2^{\mathrm{a}}$ \\
\hline $\mathrm{Ag}_{2} \mathrm{Se} \rightarrow 2 \mathrm{Ag}+\mathrm{Se}$ & $107.6 \pm 4.0^{\mathrm{a}}$ \\
\hline $\mathrm{Ag}_{2} \mathrm{Te} \rightarrow 2 \mathrm{Ag}+\mathrm{Te}$ & $\approx 96.9 \pm 3.2^{\mathrm{c}}$ \\
\hline $\mathrm{AgCN} \rightarrow \mathrm{Ag}+\mathrm{CN}$ & $86.9 \pm 2.5^{\mathrm{a}}$ \\
\hline $\mathrm{AgNC} \rightarrow \mathrm{Ag}+\mathrm{CN}$ & $73.6 \pm 2.6^{\mathrm{a}}$ \\
\hline $\mathrm{AgNH}_{2} \rightarrow \mathrm{Ag}+\mathrm{NH}_{2}$ & $44.4 \pm 2.5^{\mathrm{a}}$ \\
\hline $\mathrm{AgPH}_{2} \rightarrow \mathrm{Ag}+\mathrm{PH}_{2}$ & $\approx 55.2 \pm 2.9^{\mathrm{c}}$ \\
\hline $\mathrm{AgAsH}_{2} \rightarrow \mathrm{Ag}+\mathrm{AsH}_{2}$ & $\approx 45.3 \pm 2.8^{\mathrm{c}}$ \\
\hline $\mathrm{AgCH}_{3} \rightarrow \mathrm{Ag}+\mathrm{CH}_{3}$ & $45.6 \pm 2.6^{\mathrm{a}}$ \\
\hline $\mathrm{AgSiH}_{3} \rightarrow \mathrm{Ag}+\mathrm{SiH}_{3}$ & $\approx 58.5 \pm 3.0^{\mathrm{c}}$ \\
\hline $\mathrm{AgGeH}_{3} \rightarrow \mathrm{Ag}+\mathrm{GeH}_{3}$ & $\approx 52.3 \pm 7.7^{\mathrm{c}}$ \\
\hline $\mathrm{AgNO}_{2} \rightarrow \mathrm{Ag}+\mathrm{NO}_{2}$ & $42.8 \pm 2.6^{\mathrm{a}}$ \\
\hline $\mathrm{AgOH} \rightarrow \mathrm{Ag}+\mathrm{OH}$ & $57.8 \pm 2.5^{\mathrm{a}}$ \\
\hline $\mathrm{AgSH} \rightarrow \mathrm{Ag}+\mathrm{SH}$ & $58.1 \pm 4.6^{\mathrm{a}}$ \\
\hline $\mathrm{AgHe}^{+} \rightarrow \mathrm{Ag}^{+}+\mathrm{He}$ & $3.2 \pm 2.5^{\mathrm{a}}$ \\
\hline $\mathrm{AgNe}^{+} \rightarrow \mathrm{Ag}^{+}+\mathrm{Ne}$ & $3.7 \pm 2.5^{\mathrm{a}}$ \\
\hline $\mathrm{AgAr}^{+} \rightarrow \mathrm{Ag}^{+}+\mathrm{Ar}$ & $9.6 \pm 2.5^{\mathrm{a}}$ \\
\hline $\mathrm{AgKr}^{+} \rightarrow \mathrm{Ag}^{+}+\mathrm{Kr}$ & $13.4 \pm 2.5^{\mathrm{a}}$ \\
\hline
\end{tabular}




\begin{tabular}{cc}
\hline $\mathrm{AgXe}^{+} \rightarrow \mathrm{Ag}^{+}+\mathrm{Xe}$ & $19.2 \pm 2.5^{\mathrm{a}}$ \\
$\mathrm{AgRn}^{+} \rightarrow \mathrm{Ag}^{+}+\mathrm{Rn}$ & $22.0 \pm 2.5^{\mathrm{a}}$ \\
$\mathrm{AgBe}^{+} \rightarrow \mathrm{Ag}^{+}+\mathrm{Be}$ & $42.2 \pm 3.7^{\mathrm{a}}$ \\
$\mathrm{AgMg}^{+} \rightarrow \mathrm{Ag}^{+}+\mathrm{Mg}$ & $52.6 \pm 2.9^{\mathrm{a}}$ \\
$\mathrm{AgCa}^{+} \rightarrow \mathrm{Ag}+\mathrm{Ca}^{+}$ & $44.4 \pm 2.7^{\mathrm{a}}$ \\
$\mathrm{AgSr}^{+} \rightarrow \mathrm{Ag}+\mathrm{Sr}^{+}$ & $41.0 \pm 2.9^{\mathrm{a}}$ \\
$\mathrm{AgZn}^{+} \rightarrow \mathrm{Ag}^{+}+\mathrm{Zn}$ & $35.8 \pm 2.7^{\mathrm{a}}$ \\
$\mathrm{AgCd}^{+} \rightarrow \mathrm{Ag}^{+}+\mathrm{Cd}$ & $37.5 \pm 2.7^{\mathrm{a}}$ \\
$\mathrm{AgHg}^{+} \rightarrow \mathrm{Ag}^{+}+\mathrm{Hg}$ & $28.0 \pm 2.5^{\mathrm{a}}$
\end{tabular}

\footnotetext{
${ }^{a}$ An uncertainty is calculated as the sum of absolute values of uncertainties of gas phase formation enthalpies in Table 2/3 and corresponding reference heats of formation in Tables S1 and S5. ${ }^{b}$ The value is entirely based on experimental reference heats of formation in Tables S1 and S5. ${ }^{\mathrm{c}}$ Value is of low accuracy due to poorly defined reference data.
}

\section{Conclusions}

Proven to be reasonably accurate, a reduced version of reaction-based Feller-Peterson-Dixon (FPD) approach has been utilized to derive gas phase heats of formation and absolute entropies of 100 silver species to significantly enrich silver thermochemistry. The approach includes correlation of sub-valence electrons, complete basis set (CBS) extrapolated aug-cc-pwCVTZ/augcc-pwCVQZ DLPNO-CCSD(T) energies and DFT geometries and harmonic frequencies. Comparison of thus predicted heats of formation and entropies with their scarce experimental and theoretical counterparts (10 molecules in total) indicated, in general, good quality of the literature data, despite large experimental uncertainties given for some of them. Further, a combination of solid-state enthalpies and entropies available for four compounds with predicted in this work gas phase data, allowed us derivation of thermodynamic functions of sublimation. Finally, merging of 
predicted silver gas phase data with accurate experimental formation enthalpies of some neutrals and positive ions resulted in 33 silver bond strengths of high reliability. Obtained data are relevant for developing concepts of silver chemistry and thermochemistry. Apart from that, these can be used to train and test semiempirical models of quantum and computational chemistry, in particular becoming popular neutral network based approaches. ${ }^{152-160}$

\section{ASSOCIATED CONTENT}

Supporting Information. Tables with the reference heats of formation and gas phase enthalpies of reactions composed to derive the target heats of formation, Cartesian coordinates ( $\AA$ ), DLPNO$\operatorname{CCSD}(\mathrm{T})$ energies with different basis sets, DKH DLPNO-CCSD(T)/aug-cc-pwCVTZ-DK energies of species related to Table 2, CCSD(T)/aug-cc-pwCVTZ(-PP) energies of species related to Table $2, T_{1}$ and $T_{2}$ diagnostic values, enthalpic corrections and absolute gas phase entropies calculated via rigid rotor, ideal gas, harmonic oscillator. This material is available free of charge via the Internet at http://pubs.acs.org.

\section{AUTHOR INFORMATION}

\section{Corresponding Author}

E-mail: Yury.Minenkov@phystech.edu

\section{Funding Sources}

The research reported in this publication was supported by funding from the Government of the Russian Federation (Agreement № 074-02-2018-286). L.C. gratefully acknowledges the financial support from King Abdullah University of Science and Technology (KAUST). V.S. gratefully acknowledges the financial support from Ministry of Science and Education of the Russian 
Federation (project no. 4.3232.2017/4.6). For computer time, this research used the resources of the Supercomputing Laboratory at King Abdullah University of Science and Technology (KAUST) in Thuwal, Saudi Arabia.

\section{Notes}

The authors declare no competing financial interest.

\section{ACKNOWLEDGMENT}

We gratefully acknowledge Prof. J. J. P. Stewart, Stewart Computational Chemistry, Colorado Springs, USA for helpful discussions.

\section{REFERENCES}

(1) Li, Z.; He, C. Recent Advances in Silver-Catalyzed Nitrene, Carbene, and Silylene-Transfer Reactions. European J. Org. Chem. 2006, 2006 (19), 4313-4322. https://doi.org/10.1002/ejoc.200500602.

(2) Silver in Organic Chemistry; Harmata, M., Ed.; John Wiley \& Sons: Hoboken, N.J., 2010.

(3) Tani, T. A Review of the Mechanism of Photographic Sensitivity and Roles of Silver Clusters. J. Imaging Sci. Technol. 2004, 48 (3), 278-284.

(4) Tominaga, J. The Application of Silver Oxide Thin Films to Plasmon Photonic Devices. $J$. Physics-Condensed Matter 2003, 15 (25), R1101-R1122. https://doi.org/10.1088/0953$8984 / 15 / 25 / 201$.

(5) Eachus, R. S.; Marchetti, A. P.; Muenter, A. A. The Photophysics of Silver Halide Imaging Materials. Аnnu. Rev. Phys. Chem. 1999, 50, 117-144. https://doi.org/10.1146/annurev.physchem.50.1.117. 
(6) Sushkevich, V. L.; Ivanova, I. I.; Taarning, E. Mechanistic Study of Ethanol Dehydrogenation over Silica-Supported Silver. ChemCatChem 2013, 5 (8), 2367-2373. https://doi.org/10.1002/cctc.201300033.

(7) Churakov, A. V; Efimenko, I. A.; Erofeeva, O. S.; Kuzmina, L. G.; Ivanova, N. A. Unprecedented Polymeric Trifluoroacetate Palladium-Silver Complexes: pi-Complex with $\eta^{2}$ - and $\eta^{4}$-Coordinated Toluene, as Well as a Unique Seventeen Nuclear Palladium-Silver Trifluoroacetate. Mendeleev Commun. 2016, $26 \quad$ (4), 295-297. https://doi.org/10.1016/j.mencom.2016.07.009.

(8) Munoz, M. P. Silver and Platinum-Catalysed Addition of O-H and N-H Bonds to Allenes. Chem. Soc. Rev. 2014, 43 (9), 3164-3183. https://doi.org/10.1039/c3cs60408j.

(9) Schmidbaur, H.; Schier, A. Silver-Free Gold(I) Catalysts for Organic Transformations. Zeitschrift Fur Naturforsch. Sect. B-a J. Chem. Sci. 2011, 66 (4), 329-350. https://doi.org/10.5560/ZNB.2011.66b0329.

(10) Belmont, P.; Parker, E. Silver and Gold Catalysis for Cycloisomerization Reactions. European J. Org. Chem. 2009, No. 35, 6075-6089. https://doi.org/10.1002/ejoc.200900790.

(11) Sushkevich, V. L.; Ivanova, I. I. Ag-Promoted ZrBEA Zeolites Obtained by Post-Synthetic Modification for Conversion of Ethanol to Butadiene. ChemSusChem 2016, 9 (16), 22162225. https://doi.org/10.1002/cssc.201600572.

(12) Sushkevich, V. L.; Ivanova, I. I. Mechanistic Study of Ethanol Conversion into Butadiene over Silver Promoted Zirconia Catalysts. Appl. Catal. B-Environmental 2017, 215, 36-49. https://doi.org/10.1016/j.apcatb.2017.05.060.

(13) Koretsky, G. M.; Knickelbein, M. B. The Reactions of Silver Clusters with Ethylene and 
Ethylene Oxide: Infrared and Photoionization Studies of $\mathrm{Ag}_{\mathrm{n}}\left(\mathrm{C}_{2} \mathrm{H}_{4}\right)_{\mathrm{m}}, \mathrm{Ag}_{\mathrm{n}}\left(\mathrm{C}_{2} \mathrm{H}_{4} \mathrm{O}\right)_{\mathrm{m}}$ and Their Deuterated Analogs. J. Chem. Phys. 1997, 107 (24), 10555-10566. https://doi.org/10.1063/1.474219.

(14) Bernhardt, T. M. Gas-Phase Kinetics and Catalytic Reactions of Small Silver and Gold $\begin{array}{llllllll}\text { Clusters. Int. J. } & \text { Mass } & \text { Spectrom. 2005, } 243 & \text { (1), } & \text { 1-29. }\end{array}$ https://doi.org/10.1016/j.ijms.2004.12.015.

(15) Millar, G. J.; Collins, M. Industrial Production of Formaldehyde Using Polycrystalline Silver Catalyst. Ind. Eng. Chem. Res. 2017, 56 (33), 9247-9265. https://doi.org/10.1021/acs.iecr.7b02388.

(16) Fang, G. C.; Bi, X. H. Silver-Catalysed Reactions of Alkynes: Recent Advances. Chem. Soc. Rev. 2015, 44 (22), 8124-8173. https://doi.org/10.1039/c5cs00027k.

(17) Lo, V. K. Y.; Chan, A. O. Y.; Che, C. M. Gold and Silver Catalysis: From Organic Transformation to Bioconjugation. Org. Biomol. Chem. 2015, 13 (24), 6667-6680. https://doi.org/10.1039/c5ob00407a.

(18) Wen, C.; Yin, A. Y.; Dai, W. L. Recent Advances in Silver-Based Heterogeneous Catalysts for Green Chemistry Processes. Appl. Catal. B-Environmental 2014, 160, 730-741. https://doi.org/10.1016/j.apcatb.2014.06.016.

(19) Verma, P.; Maheshwari, S. K. Applications of Silver Nanoparticles in Diverse Sectors. Int. J. Nano Dimens. 2019, 10 (1), 18-36.

(20) Smarzewska, S.; Metelka, R.; Bas, B.; Vytras, K. Recent Applications of Silver Amalgam Electrodes for Analysis of Pharmaceuticals and Vitamins. Curr. Med. Chem. 2018, 25 (33), 4138-4151. https://doi.org/10.2174/0929867324666170920143245.

(21) Khatoon, U. T.; Rao, G.; Mantravadi, K. M.; Oztekin, Y. Strategies to Synthesize Various 
Nanostructures of Silver and Their Applications - a Review. Rsc Adv. 2018, 8 (35), 1973919753. https://doi.org/10.1039/c8ra00440d.

(22) Chase Jr., M. W. NIST-JANAF Themochemical Tables, Fourth Edition. J. Phys. Chem. Ref. Data 1998, Monograph, 1-1951.

(23) NIST Chemistry Webbook, NIST Standard Reference Database Number 69, Eds. P.J. Linstrom and W.G. Mallard, National Institute of Standards and Technology, Gaithersburg MD, 20899, Http://Webbook.Nist.Gov, (Retrieved December 27, 2017). 2016.

(24) NIST Chemistry WebBook, NIST Standard Reference Database Number 69; Linstrom, P. J., Mallard, W. G., Eds.; National Institute of Standards and Technology: Gaithersburg MD, 20899, 2005. https://doi.org/citeulike-article-id:3211271.

(25) Gurvich, L. V; Karachevtsev, G. V; Kondrat'ev, V. N.; Lebedev, J. A.; Medvedev, V. A.; Potapov, V. K.; Khodeev, Y. S. Bond Dissociation Enrgies, Ionization Potentials and Electron Affinities; Nauka, Moscow, 1974.

(26) Gurvich, L. V; Veyts, I.; Alock, C. B. Thermodynamic Properties Of Individual Substances, 4th ed; Hemisphere: New York, 1989.

(27) Glushko, V. P.; Yungman, V. S.; Medvedev, V. A.; Gurvich, L. V. Thermal Constants of Substances: An Eight-Volume Set Comprising Ten Parts. New York; Wallingford (GB) : Begell House ; New York ; Chichester ; Weinheim : J. Wiley \& Sons 1999.

(28) Database Thermal Constants of Substances Http://Www.Chem.Msu.Ru/Cgi-Bin/Tkv.Pl.

(29) Krasnov, K. S. Molecular Constants of Inorganic Compounds, Reference Book; Khimia: Leningrad, 1979.

(30) Goos, E.; Burcat, A.; Ruscic, B. No Title. Ideal Gas Thermochem. Database with Updat. from Act. Thermochem. Tables, release date 26.01.2011. 
http//garfield.chem.elte.hu/Burcat/burcat.html 2011.

(31) National Research Council. 1999. Impact of Advances in Computing and Communications Technologies on Chemical Science and Technology: Report of a Workshop; Washington, DC: The National Academies Press. doi:https://doi.org/10.17226/9591. https://doi.org/doi:10.17226/9591.

(32) Dixon, D. A.; Feller, D.; Peterson, K. A. Chapter One - A Practical Guide to Reliable First Principles Computational Thermochemistry Predictions Across the Periodic Table. In Annual Reports in Computational Chemistry; Ralph, A. W., Ed.; Elsevier, 2012; Vol. Volume 8, pp 1-28. https://doi.org/http://dx.doi.org/10.1016/B978-0-444-59440-2.000016.

(33) Suntsova, M. A.; Dorofeeva, O. V. Use of G4 Theory for the Assessment of Inaccuracies in Experimental Enthalpies of Formation of Aromatic Nitro Compounds. J. Chem. Eng. Data 2016, 61 (1), 313-329. https://doi.org/10.1021/acs.jced.5b00558.

(34) Dorofeeva, O. V; Filimonova, M. A. Self-Consistent Enthalpies of Formation of Adamantanes by Isodesmic Reaction Network. J. Chem. Thermodyn. 2018, 126, 31-37. https://doi.org/10.1016/j.jct.2018.06.016.

(35) Dixon, D. A.; Smart, B. E. Numerical Simulation of Molecular Systems. The Determination of Thermochemical Properties. Chem. Eng. Commun. 1990, 98 (1), 173-185.

(36) Peterson, K. A.; Feller, D.; Dixon, D. A. Chemical Accuracy in Ab Initio Thermochemistry and Spectroscopy: Current Strategies and Future Challenges. Theor. Chem. Acc. 2012, 131 (1), 1-20. https://doi.org/10.1007/s00214-011-1079-5.

(37) Karton, A. A Computational Chemist's Guide to Accurate Thermochemistry for Organic Molecules. Wiley Interdiscip. Rev. Mol. Sci. 2016, 6 (3), 292-310. 
https://doi.org/10.1002/wcms.1249.

(38) Dorofeeva, O. V; Suntsova, M. A. Enthalpies of Formation of Nitromethane and Nitrobenzene: Theory vs Experiment. J. Chem. Thermodyn. 2013, 58, 221-225. https://doi.org/10.1016/j.jct.2012.11.008.

(39) Dorofeeva, O. V; Ryzhova, O. N. Gas-Phase Enthalpies of Formation and Enthalpies of Sublimation of Amino Acids Based on Isodesmic Reaction Calculations. J. Phys. Chem. A 2014, 118 (19), 3490-3502. https://doi.org/10.1021/jp501357y.

(40) Suntsova, M. A.; Dorofeeva, O. V. Use of G4 Theory for the Assessment of Inaccuracies in Experimental Enthalpies of Formation of Aliphatic Nitro Compounds and Nitramines. $J$. Chem. Eng. Data 2014, 59 (9), 2813-2826. https://doi.org/10.1021/je500440y.

(41) Verevkin, S. P.; Emel'yanenko, V. N.; Diky, V.; Dorofeeva, O. V. Enthalpies of Formation of Nitromethane and Nitrobenzene: New Experiments vs. Quantum Chemical Calculations. J. Chem. Thermodyn. 2014, 73, 163-170. https://doi.org/10.1016/j.jct.2013.12.013.

(42) Dorofeeva, O. V; Ryzhova, O. N. Enthalpy of Formation and O-H Bond Dissociation Enthalpy of Phenol: Inconsistency between Theory and Experiment. J. Phys. Chem. A 2016, 120 (15), 2471-2479. https://doi.org/10.1021/acs.jpca.6b02233.

(43) Boese, A. D.; Oren, M.; Atasoylu, O.; Martin, J. M. L.; Kállay, M.; Gauss, J. W3 Theory: Robust Computational Thermochemistry in the $\mathrm{kJ} / \mathrm{mol}$ Accuracy Range. J. Chem. Phys. 2004, $120(9), 4129-4141$.

(44) Karton, A.; Rabinovich, E.; Martin, J. M. L.; Ruscic, B. W4 Theory for Computational Thermochemistry: In Pursuit of Confident Sub-kJ/mol Predictions. J. Chem. Phys. 2006, 125 (14), 144108. https://doi.org/10.1063/1.2348881.

(45) Curtiss, L. A.; Redfern, P. C.; Raghavachari, K. Gaussian-4 Theory. J. Chem. Phys. 2007, 
126 (8). https://doi.org/10.1063/1.2436888.

(46) DeYonker, N. J.; Cundari, T. R.; Wilson, A. K. The Correlation Consistent Composite Approach (CcCA): An Alternative to the Gaussian-n Methods. J. Chem. Phys. 2006, 124 (11), 114104. https://doi.org/10.1063/1.2173988.

(47) Feller, D.; Peterson, K. A.; Dixon, D. A. A Survey of Factors Contributing to Accurate Theoretical Predictions of Atomization Energies and Molecular Structures. J. Chem. Phys. 2008, 129 (20), 204105. https://doi.org/10.1063/1.3008061.

(48) Feller, D.; Peterson, K. A.; Dixon, D. A. Further Benchmarks of a Composite, Convergent, Statistically Calibrated Coupled-Cluster-Based Approach for Thermochemical and Spectroscopic Studies. Mol. Phys. 2012, $110 \quad$ (19-20), 2381-2399. https://doi.org/10.1080/00268976.2012.684897.

(49) Raghavachari, K.; Trucks, G. W.; Pople, J. A.; Headgordon, M. A Fifth-Order Perturbation Comparison of Electron Correlation Theories. Chem. Phys. Lett. 1989, 157 (6), 479-483. https://doi.org/10.1016/s0009-2614(89)87395-6.

(50) Neese, F.; Hansen, A.; Liakos, D. G. Efficient and Accurate Approximations to the Local Coupled Cluster Singles Doubles Method Using a Truncated Pair Natural Orbital Basis. J. Chem. Phys. 131 (6), 64103. https://doi.org/10.1063/1.3173827.

(51) Hampel, C.; Werner, H. J. Local Treatment of Electron Correlation in Coupled Cluster Theory. J. Chem. Phys. 1996, 104 (16), 6286-6297. https://doi.org/10.1063/1.471289.

(52) Schütz, M.; Werner, H. J. Local Perturbative Triples Correction (T) with Linear Cost Scaling. Chem. Phys. Lett. 2000, 318 (4-5), 370-378. https://doi.org/10.1016/s00092614(00)00066-x.

(53) Schutz, M.; Werner, H. J. Low-Order Scaling Local Electron Correlation Methods. IV. 
Linear Scaling Local Coupled-Cluster (LCCSD). J. Chem. Phys. 2001, 114 (2), 661-681.

(54) Riplinger, C.; Neese, F. An Efficient and near Linear Scaling Pair Natural Orbital Based

Local Coupled Cluster Method. J. Chem. Phys. 138 (3), 34106. https://doi.org/10.1063/1.4773581.

(55) Riplinger, C.; Sandhoefer, B.; Hansen, A.; Neese, F. Natural Triple Excitations in Local Coupled Cluster Calculations with Pair Natural Orbitals. J. Chem. Phys. 139 (13), 134101. https://doi.org/10.1063/1.4821834.

(56) Riplinger, C.; Pinski, P.; Becker, U.; Valeev, E. F.; Neese, F. Sparse Maps-A Systematic Infrastructure for Reduced-Scaling Electronic Structure Methods. II. Linear Scaling Domain Based Pair Natural Orbital Coupled Cluster Theory. J. Chem. Phys. 2016, 144 (2), 24109. https://doi.org/10.1063/1.4939030.

(57) Hättig, C.; Weigend, F. CC2 Excitation Energy Calculations on Large Molecules Using the Resolution of the Identity Approximation. J. Chem. Phys. 2000, 113 (13), 5154-5161.

(58) Werner, H.-J.; Schütz, M. An Efficient Local Coupled Cluster Method for Accurate Thermochemistry of Large Systems. J. Chem. Phys. 135 (14), 144116. https://doi.org/10.1063/1.3641642.

(59) Liakos, D. G.; Hansen, A.; Neese, F. Weak Molecular Interactions Studied with Parallel Implementations of the Local Pair Natural Orbital Coupled Pair and Coupled Cluster Methods. J. Chem. Theory Comput. 2011, 7 (1), 76-87. https://doi.org/10.1021/ct100445s.

(60) Minenkov, Y.; Wang, H.; Wang, Z.; Sarathy, S. M.; Cavallo, L. Heats of Formation of Medium-Sized Organic Compounds from Contemporary Electronic Structure Methods. J. Chem. Theory Comput. 2017, 13 (8). https://doi.org/10.1021/acs.jctc.7b00335.

(61) Paulechka, E.; Kazakov, A. Efficient DLPNO-CCSD(T)-Based Estimation of Formation 
Enthalpies for C-, H-, O-, and N-Containing Closed-Shell Compounds Validated Against Critically Evaluated Experimental Data. J. Phys. Chem. A 2017, 121, 4379-4387. https://doi.org/10.1021/acs.jpca.7b03195.

(62) Paulechka, E.; Kazakov, A. Efficient Estimation of Formation Enthalpies for Closed-Shell Organic Compounds with Local Coupled-Cluster Methods. J. Chem. Theory Comput. 2018, $14(11), 5920-5932$.

(63) Minenkov, Y.; Sliznev, V. V.; Cavallo, L. Accurate Gas Phase Formation Enthalpies of Alloys and Refractories Decomposition Products. Inorg. Chem. 2017, 56 (3). https://doi.org/10.1021/acs.inorgchem.6b02441.

(64) Minenkov, Y.; Bistoni, G.; Riplinger, C.; Auer, A. A.; Neese, F.; Cavallo, L. Pair Natural Orbital and Canonical Coupled Cluster Reaction Enthalpies Involving Light to Heavy Alkali and Alkaline Earth Metals: The Importance of Sub-Valence Correlation. Phys. Chem. Chem. Phys. 2017, 19 (14). https://doi.org/10.1039/c7cp00836h.

(65) Koch, W.; Holthausen, M. C.; Baerends, E. J. A Chemist's Guide to Density Functional Theory; WILEY-VCH Verlag GmbH: Weinheim.

(66) Hohenberg, P.; Kohn, W. INHOMOGENEOUS ELECTRON GAS. Phys. Rev. B 1964, 136 (3B), B864. https://doi.org/10.1103/PhysRev.136.B864.

(67) Stewart, J. J. P. Semiempirical Molecular Orbital Methods. In Reviews in Computational Chemistry; John Wiley \& Sons, Inc., 2007; pp 45-81. https://doi.org/10.1002/9780470125786.ch2.

(68) Thiel, W. Semiempirical Quantum-Chemical Methods. Wiley Interdiscip. Rev. Mol. Sci. 2014, 4 (2), 145-157. https://doi.org/10.1002/wcms.1161.

(69) Becke, A. D. Density-Functional Exchange-Energy Approximation with Correct 
Asymptotic Behavior. Phys. Rev. A 1988, 38 (6), 3098-3100. https://doi.org/10.1103/PhysRevA.38.3098.

(70) Miehlich, B.; Savin, A.; Stoll, H.; Preuss, H. Results Obtained with the Correlation Energy Density Functionals of Becke and Lee, Yang and Parr. Chem. Phys. Lett. 1989, 157 (3), 200-206. https://doi.org/10.1016/0009-2614(89)87234-3.

(71) Lee, C. T.; Yang, W. T.; Parr, R. G. Development of the Colle-Salvetti Correlation-Energy Formula into a Functional of the Electron Density. Phys. Rev. B 1988, 37 (2), 785-789. https://doi.org/10.1103/PhysRevB.37.785.

(72) Minenkov, Y.; Chermak, E.; Cavallo, L. Troubles in the Systematic Prediction of Transition Metal Thermochemistry with Contemporary Out-of-the-Box Methods. J. Chem. Theory Comput. 2016, 12 (4). https://doi.org/10.1021/acs.jctc.5b01163.

(73) Xu, X.; Zhang, W.; Tang, M.; Truhlar, D. G. Do Practical Standard Coupled Cluster Calculations Agree Better than Kohn-Sham Calculations with Currently Available Functionals When Compared to the Best Available Experimental Data for Dissociation Energies of Bonds to 3d Transition Metals? J. Chem. Theory Comput. 2015, 11 (5), 20362052. https://doi.org/10.1021/acs.jctc.5b00081.

(74) Moltved, K. A.; Kepp, K. P. Chemical Bond Energies of 3d Transition Metals Studied by Density Functional Theory. J. Chem. Theory Comput. 2018, 14 (7), 3479-3492.

(75) Aoto, Y. A.; de Lima Batista, A. P.; Köhn, A.; de Oliveira-Filho, A. G. S. How To Arrive at Accurate Benchmark Values for Transition Metal Compounds: Computation or Experiment? J. Chem. Theory Comput. 2017, 13 (11), 5291-5316.

(76) Determan, J. J.; Poole, K.; Scalmani, G.; Frisch, M. J.; Janesko, B. G.; Wilson, A. K. Comparative Study of Nonhybrid Density Functional Approximations for the Prediction of 
3d Transition Metal Thermochemistry. J. Chem. Theory Comput. 2017, 13 (10), 4907-4913.

(77) Cheng, L.; Gauss, J.; Ruscic, B.; Armentrout, P. B.; Stanton, J. F. Bond Dissociation Energies for Diatomic Molecules Containing 3d Transition Metals: Benchmark ScalarRelativistic Coupled-Cluster Calculations for 20 Molecules. J. Chem. Theory Comput. 2017. https://doi.org/10.1021/acs.jctc.6b00970.

(78) Fang, Z.; Vasiliu, M.; Peterson, K. A.; Dixon, D. A. Prediction of Bond Dissociation Energies/Heats of Formation for Diatomic Transition Metal Compounds: CCSD(T) Works. J. Chem. Theory Comput. 2017, 13, 1057-1066. https://doi.org/10.1021/acs.jctc.6b00971.

(79) Perdew, J. P.; Burke, K.; Ernzerhof, M. Generalized Gradient Approximation Made Simple. Phys. Rev. Lett. 1996, 77 (18), 3865-3868. https://doi.org/10.1103/PhysRevLett.77.3865.

(80) Perdew, J. P.; Burke, K.; Ernzerhof, M. Generalized Gradient Approximation Made Simple. Phys. Rev. Lett. 1997, 78 (7), 1396. https://doi.org/10.1103/PhysRevLett.78.1396.

(81) Adamo, C.; Barone, V. Exchange Functionals with Improved Long-Range Behavior and Adiabatic Connection Methods without Adjustable Parameters: The MPW and MPW1PW Models. J. Chem. Phys. 1998, 108 (2), 664-675. https://doi.org/10.1063/1.475428.

(82) Frisch, M. J.; Trucks, G. W.; Schlegel, H. B.; Scuseria, G. E.; Robb, M. A.; Cheeseman, J. R.; Scalmani, G.; Barone, V.; Mennucci, B.; Petersson, G. A.; et al. Gaussian 09. Gaussian, Inc.: Wallingford, CT, USA 2009.

(83) Waller, M. P.; Braun, H.; Hojdis, N.; Bühl, M. Geometries of Second-Row Transition Metal Complexes from Density Functional Theory. J. Chem. Theory Comput. 2007, 3 (6), 22342242. https://doi.org/10.1021/ct700178y.

(84) Bühl, M.; Reimann, C.; Pantazis, D. A.; Bredow, T.; Neese, F. Geometries of Third-Row Transition-Metal Complexes from Density Functional Theory. J. Chem. Theory Comput. 
2008, 4 (9), 1449-1459. https://doi.org/10.1021/ct800172j.

(85) Bühl, M.; Kabrede, H. Geometries of Transition Metal Complexes from Density Functional Theory. J. Chem. Theory Comput. 2006, 2 (5), 1282-1290. https://doi.org/10.1021/ct6001187.

(86) Minenkov, Y.; Cavallo, L. Ground-State Gas-Phase Structures of Inorganic Molecules Predicted by Density Functional Theory Methods. ACS Omega 2017, 2 (11), 8373-8387. https://doi.org/10.1021/acsomega.7b01203.

(87) Krasnov, K. S. Physical Chemistry; "Vyshaya Shkola": Moscow NV - 2, 2001; Vol. 1.

(88) Dill, K.; Bromberg, S. Molecular Driving Forces: Statistical Thermodynamics in Biology, Chemistry, Physics, and Nanoscience; Taylor \& Francis Group, 2010.

(89) Merrick, J. P.; Moran, D.; Radom, L. An Evaluation of Harmonic Vibrational Frequency Scale Factors. J. Phys. Chem. A 2007, 111 (45), 11683-11700. https://doi.org/10.1021/jp073974n.

(90) Kesharwani, M. K.; Brauer, B.; Martin, J. M. L. Frequency and Zero-Point Vibrational Energy Scale Factors for Double-Hybrid Density Functionals (and Other Selected Methods): Can Anharmonic Force Fields Be Avoided? J. Phys. Chem. A 2015, 119 (9), 1701-1714. https://doi.org/10.1021/jp508422u.

(91) Dunning, T. H. Gaussian-Basis Sets for Use in Correlated Molecular Calculations .1. The Atoms Boron through Neon and Hydrogen. J. Chem. Phys. 1989, 90 (2), 1007-1023. https://doi.org/10.1063/1.456153.

(92) Woon, D. E.; Dunning, T. H. Gaussian-Basis Sets for Use in Correlated Molecular Calculations .3. The Atoms Aluminum through Argon. J. Chem. Phys. 1993, 98 (2), 13581371. https://doi.org/10.1063/1.464303. 
(93) Peterson, K. A. Systematically Convergent Basis Sets with Relativistic Pseudopotentials. I. Correlation Consistent Basis Sets for the Post-d Group 13-15 Elements. J. Chem. Phys. 2003, 119 (21), 11099-11112. https://doi.org/10.1063/1.1622923.

(94) Peterson, K. A.; Figgen, D.; Goll, E.; Stoll, H.; Dolg, M. Systematically Convergent Basis Sets with Relativistic Pseudopotentials. II. Small-Core Pseudopotentials and Correlation Consistent Basis Sets for the Post-d Group 16-18 Elements. J. Chem. Phys. 2003, 119 (21), 11113-11123. https://doi.org/10.1063/1.1622924.

(95) Peterson, K. A.; Puzzarini, C. Systematically Convergent Basis Sets for Transition Metals. II. Pseudopotential-Based Correlation Consistent Basis Sets for the Group $11(\mathrm{Cu}, \mathrm{Ag}, \mathrm{Au})$ and 12 ( $\mathrm{Zn}, \mathrm{Cd}, \mathrm{Hg})$ Elements. Theor. Chem. Acc. 2005, 114 (4-5), 283-296. https://doi.org/10.1007/s00214-005-0681-9.

(96) Peterson, K. A.; Shepler, B. C.; Figgen, D.; Stoll, H. On the Spectroscopic and Thermochemical Properties of ClO, BrO, IO, and Their Anions. J. Phys. Chem. A 2006, 110 (51), 13877-13883. https://doi.org/10.1021/jp0658871.

(97) Metz, B.; Stoll, H.; Dolg, M. Small-Core Multiconfiguration-Dirac-Hartree-Fock-Adjusted Pseudopotentials for Post-d Main Group Elements: Application to PbH and PbO. J. Chem. Phys. 2000, 113 (7), 2563-2569. https://doi.org/10.1063/1.1305880.

(98) Figgen, D.; Rauhut, G.; Dolg, M.; Stoll, H. Energy-Consistent Pseudopotentials for Group 11 and 12 Atoms: Adjustment to Multi-Configuration Dirac-Hartree-Fock Data. Chem. Phys. 2005, 311 (1-2), 227-244. https://doi.org/10.1016/j.chemphys.2004.10.005.

(99) Neese, F. Software Update: The ORCA Program System, Version 4.0. Wiley Interdiscip. Rev. Mol. Sci. 2018, 8 (1). https://doi.org/10.1002/wcms.1327.

(100) Liakos, D. G.; Sparta, M.; Kesharwani, M. K.; Martin, J. M. L.; Neese, F. Exploring the 
Accuracy Limits of Local Pair Natural Orbital Coupled-Cluster Theory. J. Chem. Theory Comput. 2015, 11 (4), 1525-1539. https://doi.org/10.1021/ct501129s.

(101) Bistoni, G.; Riplinger, C.; Minenkov, Y.; Cavallo, L.; Auer, A. A.; Neese, F. Treating Subvalence Correlation Effects in Domain Based Pair Natural Orbital Coupled Cluster Calculations: An Out-of-the-Box Approach. J. Chem. Theory Comput. 2017, 13 (7). https://doi.org/10.1021/acs.jctc.7b00352.

(102) Rodgers, M. T.; Armentrout, P. B. A Critical Evaluation of the Experimental and Theoretical Determination of Lithium Cation Affinities. Int. J. Mass Spectrom. 2007, 267 (1), 167-182. https://doi.org/https://doi.org/10.1016/j.ijms.2007.02.034.

(103) Bauschlicher, C. W.; Melius, C. F.; Allendorf, M. D. Gallium Compounds, a Possible Problem for the G2 Approaches. J. Chem. Phys. 1999, 110 (4), 1879-1881. https://doi.org/10.1063/1.477851.

(104) Schulz, A.; Smith, B. J.; Radom, L. Heats of Formation of Alkali and Alkaline Earth Oxides and Hydroxides: Some Dramatic Failures of the G2 Method. J. Phys. Chem. A 1999, 103 (37), 7522-7527. https://doi.org/10.1021/jp991577+.

(105) Sullivan, M. B.; Iron, M. A.; Redfern, P. C.; Martin, J. M. L.; Curtiss, L. A.; Radom, L. Heats of Formation of Alkali Metal and Alkaline Earth Metal Oxides and Hydroxides: Surprisingly Demanding Targets for High-Level Ab Initio Procedures. J. Phys. Chem. A 2003, 107 (29), 5617-5630. https://doi.org/10.1021/jp034851f.

(106) Austin, A. J.; Frisch, M. J.; Montgomery, J. A.; Petersson, G. A. An Overlap Criterion for Selection of Core Orbitals. Theor. Chem. Acc. 2002, 107 (3), 180-186. https://doi.org/10.1007/s00214-001-0310-1.

(107) Ranasinghe, D. S.; Frisch, M. J.; Petersson, G. A. Core-Core and Core-Valence Correlation 
Energy Atomic and Molecular Benchmarks for Li through Ar. J. Chem. Phys. 2015, 143 (21), 214110. https://doi.org/10.1063/1.4935972.

(108) Bartlett, R. J.; Musial, M. Coupled-Cluster Theory in Quantum Chemistry. Rev. Mod. Phys. 2007, 79 (1), 291-352. https://doi.org/10.1103/RevModPhys.79.291.

(109) Peterson, K. A.; Dunning, T. H. Accurate Correlation Consistent Basis Sets for Molecular Core-Valence Correlation Effects: The Second Row Atoms Al-Ar, and the First Row Atoms B-Ne Revisited. J. Chem. Phys. 2002, 117 (23), 10548-10560. https://doi.org/10.1063/1.1520138.

(110) Peterson, K. A.; Yousaf, K. E. Molecular Core-Valence Correlation Effects Involving the Post-d Elements Ga-Rn: Benchmarks and New Pseudopotential-Based Correlation Consistent Basis Sets. J. Chem. Phys. 2010, 133 (17), 174116. https://doi.org/10.1063/1.3503659.

(111) Stoychev, G. L.; Auer, A. A.; Neese, F. Automatic Generation of Auxiliary Basis Sets. J. Chem. Theory Comput. 2017, 13 (2), 554-562. https://doi.org/10.1021/acs.jctc.6b01041.

(112) Lee, T. J.; Taylor, P. R. A Diagnostic for Determining the Quality of Single-Reference Electron Correlation Methods. Int. J. Quantum Chem. 1989, 23, 199-207. https://doi.org/10.1002/qua.560360824.

(113) Liakos, D. G.; Neese, F. Interplay of Correlation and Relativistic Effects in Correlated

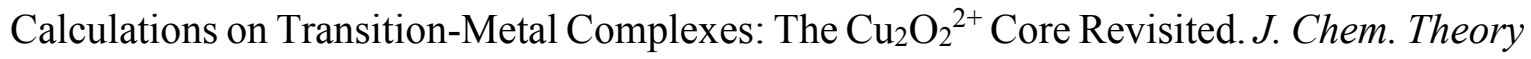
Comput. 2011, 7 (5), 1511-1523. https://doi.org/10.1021/ct1006949.

(114) Cramer, C. J.; Włoch, M.; Piecuch, P.; Puzzarini, C.; Gagliardi, L. Theoretical Models on the $\mathrm{Cu}_{2} \mathrm{O}_{2}$ Torture Track: Mechanistic Implications for Oxytyrosinase and Small-Molecule Analogues. J. Phys. Chem. A 2006, 110 (5), 1991-2004. https://doi.org/10.1021/jp056791e. 
(115) Halkier, A.; Helgaker, T.; Jørgensen, P.; Klopper, W.; Koch, H.; Olsen, J.; Wilson, A. K. Basis-Set Convergence in Correlated Calculations on $\mathrm{Ne}, \mathrm{N}_{2}$, and $\mathrm{H}_{2} \mathrm{O}$. Chem. Phys. Lett. 1998, 286 (3-4), 243-252. https://doi.org/10.1016/s0009-2614(98)00111-0.

(116) Helgaker, T.; Klopper, W.; Koch, H.; Noga, J. Basis-Set Convergence of Correlated Calculations on Water. J. Chem. Phys. 1997, 106 (23), 9639-9646.

(117) Halkier, A.; Helgaker, T.; Jørgensen, P.; Klopper, W.; Olsen, J. Basis-Set Convergence of the Energy in Molecular Hartree-Fock Calculations. Chem. Phys. Lett. 1999, 302 (5-6), 437-446. https://doi.org/10.1016/s0009-2614(99)00179-7.

(118) Hehre, W. J.; Ditchfield, R.; Radom, L.; Pople, J. A. Molecular Orbital Theory of the Electronic Structure of Organic Compounds. V. Molecular Theory of Bond Separation. $J$. Am. Chem. Soc. 1970, 92 (16), 4796-4801. https://doi.org/10.1021/ja00719a006.

(119) Ruscic, B.; Pinzon, R. E.; von Laszewski, G.; Kodeboyina, D.; Burcat, A.; Leahy, D.; Montoya, D.; Wagner, A. F. Active Thermochemical Tables: Thermochemistry for the 21st Century. In SciDAC 2005: Scientific Discovery Through Advanced Computing; Mezzacappa, A., Ed.; Journal of Physics Conference Series; 2005; Vol. 16, pp 561-570. https://doi.org/10.1088/1742-6596/16/1/078.

(120) Vasiliu, M.; Li, S. G.; Peterson, K. A.; Feller, D.; Gole, J. L.; Dixon, D. A. Structures and Heats of Formation of Simple Alkali Metal Compounds: Hydrides, Chlorides, Fluorides, Hydroxides, and Oxides for Li, Na, and K. J. Phys. Chem. A 2010, 114 (12), 4272-4281. https://doi.org/10.1021/jp911735c.

(121) Vasiliu, M.; Hill, J. G.; Peterson, K. A.; Dixon, D. A. Structures and Heats of Formation of Simple Alkaline Earth Metal Compounds II: Fluorides, Chlorides, Oxides, and Hydroxides for Ba, Sr, and Ra. J. Phys. Chem. A 2018, 122 (1), 316-327. 
(122) Medvedev, V. A.; Wagman, D. D.; Cox, J. D. CODATA Key Values for Thermodynamics; 1989.

(123) Hildenbrand, D. L.; Lau, K. H. Thermochemistry of Gaseous $\mathrm{AgCl}, \mathrm{Ag}_{3} \mathrm{Cl}_{3}$, and CuCl. High Temp. Mater. Sci. 1996, 35 (1), 11-20.

(124) Feller, D.; Peterson, K. A.; Dixon, D. A. The Impact of Larger Basis Sets and Explicitly Correlated Coupled Cluster Theory on the Feller-Peterson-Dixon Composite Method. In Annual Reports in Computational Chemistry, Volume 12; Dixon, D. A., Ed.; Elsevier Science, 2016.

(125) Bross, D. H.; Peterson, K. A. Composite Thermochemistry of Gas Phase U(VI)-Containing Molecules. J. Chem. Phys. 2014, 141 (24), 244308. https://doi.org/10.1063/1.4904721.

(126) Vasiliu, M.; Grant, D. J.; Feller, D.; Dixon, D. A. Heats of Formation of MHxCly (M = Si, P, As, Sb) Compounds and Main Group Fluorides from High Level Electronic Structure Calculations. J. Phys. Chem. A 2012, 116 (14), 3717-3727. https://doi.org/10.1021/jp2119229.

(127) Minenkov, Y.; Singstad, Å.; Occhipinti, G.; Jensen, V. R. The Accuracy of DFT-Optimized Geometries of Functional Transition Metal Compounds: A Validation Study of Catalysts for Olefin Metathesis and Other Reactions in the Homogeneous Phase. Dalton Transactions. 2012, p 5526. https://doi.org/10.1039/c2dt12232d.

(128) Stephens, S. L.; Mizukami, W.; Tew, D. P.; Walker, N. R.; Legon, A. C. Molecular Geometry of OC. - AgI Determined by Broadband Rotational Spectroscopy and Ab Initio Calculations. J. Chem. Phys. 2012, $136 \quad$ (6), 64306. https://doi.org/10.1063/1.3683221.

(129) Le Roy, R. J.; Appadoo, D. R. T.; Anderson, K.; Shayesteh, A.; Gordon, I. E.; Bernath, P. 
F. Direct-Potential-Fit Analysis of New Infrared and UV/Visible $\mathrm{A} \Sigma+{ }^{1}-\mathrm{X} \Sigma+{ }^{1}$ Emission Spectra of AgH and AgD. J. Chem. Phys. 2005, 123 (20), 204304. https://doi.org/10.1063/1.2064947.

(130) Barrow, R. F.; Clements, R. M. Rotational Analysis of the $\mathrm{A} 0^{+}, \mathrm{B} 0^{+} \leftarrow \mathrm{X}^{1} \Sigma^{+}$Systems of Gaseous AgF. Proc. R. Soc. Lond. A. Math. Phys. Sci. 1971, 322 (1549), 243-249.

(131) Krisher, L. C.; Norris, W. G. Microwave Spectrum of Silver Chloride. J. Chem. Phys. 1966, 44 (1), 391-394. https://doi.org/10.1063/1.1726476.

(132) Hoeft, J.; Lovas, F. J.; Tiemann, E.; Torring, T. Microwave Rotation Spectra of AgCl, AgBr and AgI. Z. Naturforsch. A 1971, A 26 (2), 240-244.

(133) Hoeft, J.; Nair, K. P. R. Millimeter-Wave Rotational Transitions and Molecular Constants of the Diatomic Silver Iodide. Chem. Phys. Lett. 1986, 129 (6), 538-540. https://doi.org/10.1016/0009-2614(86)80396-7.

(134) Simard, B.; Hackett, P. A.; James, A. M.; Langridge-Smith, P. R. R. The Bond Length of Silver Dimer. Chem. Phys. Lett. 1991, 186 (4), 415-422. https://doi.org/https://doi.org/10.1016/0009-2614(91)90201-J.

(135) Okabayashi, T.; Okabayashi, E. Y.; Koto, F.; Ishida, T.; Tanimoto, M. Detection of Free Monomeric Silver(I) and Gold(I) Cyanides, $\mathrm{AgCN}$ and $\mathrm{AuCN}$ : Microwave Spectra and Molecular Structure. $\quad J . \quad$ Am. $\quad$ Chem. Soc. 2009, 131 (33), 11712-11718. https://doi.org/10.1021/ja808153g.

(136) Grant Hill, J.; Mitrushchenkov, A. O.; Peterson, K. A. Ab Initio Ro-Vibrational Spectroscopy of the Group 11 Cyanides: CuCN, AgCN, and AuCN. J. Chem. Phys. 2013, 138 (13), 134314. https://doi.org/10.1063/1.4798638.

(137) Stephens, S. L.; Tew, D. P.; Walker, N. R.; Legon, A. C. H3P...AgI: Generation by Laser- 
Ablation and Characterization by Rotational Spectroscopy and Ab Initio Calculations. Phys. Chem. Chem. Phys. 2016, 18 (28), 18971-18977. https://doi.org/10.1039/C6CP03512D.

(138) Medcraft, C.; Gougoula, E.; Bittner, D. M.; Mullaney, J. C.; Blanco, S.; Tew, D. P.; Walker, N. R.; Legon, A. C. Molecular Geometries and Other Properties of H2O $\cdots A g I$ and H3N..AgI as Characterised by Rotational Spectroscopy and Ab Initio Calculations. $J$. Chem. Phys. 2017, 147 (23), 234308. https://doi.org/10.1063/1.5008744.

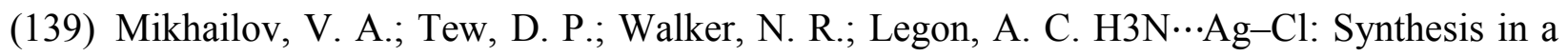
Supersonic Jet and Characterisation by Rotational Spectroscopy. Chem. Phys. Lett. 2010, 499 (1), 16-20. https://doi.org/https://doi.org/10.1016/j.cplett.2010.08.069.

(140) Lias, S. G.; Bartmess, J. E.; Liebman, J. F.; Holmes, J. L.; Levin, R. D.; Mallard, W. G. Gas-Phase Ion and Neutral Thermochemistry. J. Phys. Chem. Ref. Data 1988, 17, 1-861.

(141) Stewart, J. J. P. Http://Openmopac.Net/PM7_accuracy/Molecules.Html. Set of Individual Molecules. 2016, p The PM7 reference data.

(142) Hildenbrand, D. L.; Lau, K. H. Dissociation Energies of the Cu and Ag Monohalides and of Ni Monofluoride. J. Phys. Chem. A 2006, 110 (42), 11886-11889. https://doi.org/10.1021/jp064171n.

(143) Hildenbrand, D. L.; Lau, K. H. Composition and Thermochemistry of Silver Bromide Vapor. J. Phys. Chem. A 2005, 109 (49), 11328-11331. https://doi.org/10.1021/jp054767v.

(144) Ran, Q.; Schmude, R. W.; Gingerich, K. A.; Wilhite, D. W.; Kingcade, J. E. Dissociation Energy and Enthalpy of Formation of Gaseous Silver Dimer. J. Phys. Chem. 1993, 97 (32), 8535-8540. https://doi.org/10.1021/j100134a025.

(145) De Jong, W. A.; Harrison, R. J.; Dixon, D. A. Parallel Douglas-Kroll Energy and Gradients in NWChem: Estimating Scalar Relativistic Effects Using Douglas-Kroll Contracted Basis 
Sets. J. Chem. Phys. 2001, 114 (1), 48-53. https://doi.org/10.1063/1.1329891.

(146) Kelley, K. K.; King, E. G.; United States. Bureau of, M. Contributions to the Data on Theoretical Metallurgy: XIV. Entropies of the Elements and Inorganic Compounds; U.S. Department of the Interior, Bureau of Mines, 1961.

(147) Wagman, D. D.; al, et. Selected Values of Chemical Thermodynamics Properties. NBS, Techn. Note, N 270; 1968; Vol. Iss. 3.

(148) Pauling, L. The Nature of the Chemical Bond. IV. The Energy of Single Bonds and the Relative Electronegativity of Atoms. J. Am. Chem. Soc. 1932, 54 (9), 3570-3582.

(149) Cramer, C. J. Essentials of Computational Chemistry: Theories and Models, 2nd ed.; Wiley: Chichester, England.

(150) Jensen, F. Introduction to Computational Chemistry, 2nd ed.; Wiley: Chichester, England, 2016.

(151) Tomasi, J.; Mennucci, B.; Cammi, R. Quantum Mechanical Continuum Solvation Models. Chem. Rev. 2005, 105 (8), 2999-3093. https://doi.org/10.1021/cr9904009.

(152) Smith, J. S.; Isayev, O.; Roitberg, A. E. ANI-1: An Extensible Neural Network Potential with DFT Accuracy at Force Field Computational Cost. Chem. Sci. 2017, 8 (4), 3192-3203. https://doi.org/10.1039/c6sc05720a.

(153) Tsubaki, M.; Mizoguchi, T. Fast and Accurate Molecular Property Prediction: Learning Atomic Interactions and Potentials with Neural Networks. J. Phys. Chem. Lett. 2018, 9 (19), 5733-5741. https://doi.org/10.1021/acs.jpclett.8b01837.

(154) Margraf, J. T.; Reuter, K. Making the Coupled Cluster Correlation Energy Machine$\begin{array}{llllllll}\text { Learnable. } & \text { J. } & \text { Phys. } & \text { Chem. } & \text { A 2018, } & 122 & \text { (30), 6343-6348. }\end{array}$ https://doi.org/10.1021/acs.jpca.8b04455. 
(155) Butler, K. T.; Davies, D. W.; Cartwright, H.; Isayev, O.; Walsh, A. Machine Learning for Molecular and Materials Science. Nature 2018, 559 (7715), 547-555. https://doi.org/10.1038/s41586-018-0337-2.

(156) Popova, M.; Isayev, O.; Tropsha, A. Deep Reinforcement Learning for de Novo Drug Design. Sci. Adv. 2018, 4 (7). https://doi.org/10.1126/sciadv.aap7885.

(157) Chen, X.; Jorgensen, M. S.; Li, J.; Hammer, B. Atomic Energies from a Convolutional Neural Network. J. Chem. Theory Comput. 2018, 14 (7), 3933-3942. https://doi.org/10.1021/acs.jctc.8b00149.

(158) Schutt, K. T.; Sauceda, H. E.; Kindermans, P. J.; Tkatchenko, A.; Muller, K. R. SchNet - A Deep Learning Architecture for Molecules and Materials. J. Chem. Phys. 2018, 148 (24). https://doi.org/10.1063/1.5019779.

(159) Zhang, L. F.; Han, J. Q.; Wang, H.; Car, R.; Weinan, E. Deep Potential Molecular Dynamics: A Scalable Model with the Accuracy of Quantum Mechanics. Phys. Rev. Lett. 2018, 120 (14). https://doi.org/10.1103/PhysRevLett.120.143001.

(160) Yao, K.; Herr, J. E.; Toth, D. W.; McKintyre, R.; Parkhill, J. The TensorMol-0.1 Model Chemistry: A Neural Network Augmented with Long-Range Physics. Chem. Sci. 2018, 9 (8), 2261-2269. https://doi.org/10.1039/c7sc04934j. 


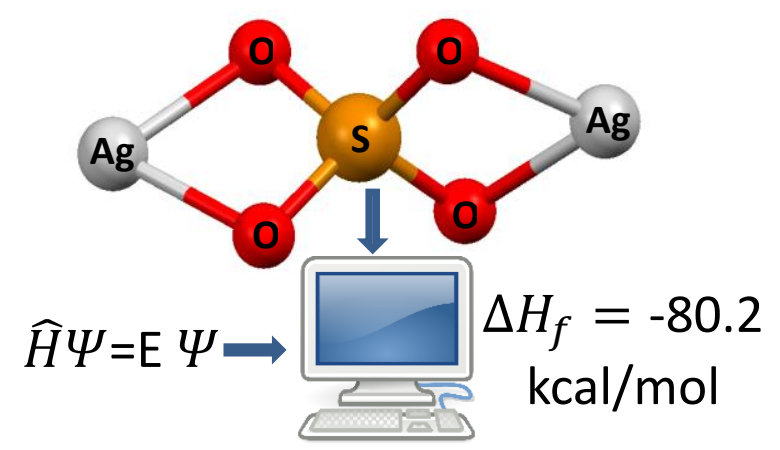

Synopsis: Domain based local pair natural orbital coupled cluster approach with single, double, and perturbative triple excitations, $\mathrm{DLPNO}-\mathrm{CCSD}(\mathrm{T})$, has been applied within a framework of a reduced version of the reaction-based Feller-Peterson-Dixon (FPD) scheme to predict heats of formation and absolute entropies of 100 silver compounds to enrich gas phase silver thermochemistry currently limited by only a few numbers from experimental measurements. 